\title{
Output Regulation of Linear Systems With Bounded Continuous Feedback
}

\author{
Tingshu Hu, Senior Member, IEEE, and Zongli Lin, Senior Member, IEEE
}

\begin{abstract}
This paper studies the classical problem of output regulation for linear systems subject to control constraint. The asymptotically regulatable region, the set of all initial conditions of the plant and the exosystem for which output regulation is possible, is characterized in terms of the null controllable region of the antistable subsystem of the plant. Continuous output regulation laws, of both state feedback type and error feedback type, are constructed from a given stabilizing state feedback law. It is shown that a stabilizing feedback law that achieves a larger domain of attraction leads to a feedback law that achieves output regulation on a larger subset of the asymptotically regulatable region. A feedback law that achieves global stabilization on the asymptotically null controllable region leads to a feedback law that achieves output regulation on the entire asymptotically regulatable region.
\end{abstract}

Index Terms-Bounded control, nonlinear control, output regulation, regulatable region.

\section{INTRODUCTION}

$\mathbf{S}$ INCE THE formulation and solution of the problem of output regulation for linear systems [4], there has been continual efforts in extending this classical control problem to various classes of nonlinear systems (see, for example, [1]-[3], [13]-[16], [18], [21], and [26]). This paper revisits the output regulation problem for linear systems with bounded controls.

There has been considerable research on the problem of stabilization and output regulation of linear systems subject to control constraints. The problem of stabilization involves the issues of the characterization of null controllable region (or, asymptotically null controllable region), the set of all initial conditions that can be driven to the origin by constrained controls in some finite time (or, asymptotically), and the construction of feedback laws that achieve stabilization on the entire or a large portion of the asymptotically null controllable region. Recent years have witnessed extensive research that addresses these issues. In particular, for an open-loop system that is stabilizable and has all its poles in the closed left-half plane, it was established in [22] and $[23]$ that the asymptotically null controllable region is the entire state space if the constrained control set contains the origin in its interior. For this reason, a linear system that is stabilizable in the usual linear sense and has all its poles in the closed

Manuscript received July 30, 2003; revised April 16, 2004. Recommended by Associate Editor J. Huang. This work was supported in part by the National Science Foundation under Grant CMS-0324329.

T. Hu was with the Charles L. Brown Department of Electrical and Computer Engineering, University of Virginia, Charlottesville, VA 22904-4743 USA. He is now with the Department of Electrical and Computer Engineering, University of California, Santa Barbara, CA 93106 USA.

Z. Lin is with the Charles L. Brown Department of Electrical and Computer Engineering, University of Virginia, Charlottesville, VA 22904-4743 USA (e-mail: z15y@virginia.edu).

Digital Object Identifier 10.1109/TAC.2004.837591 left-half plane is said to be asymptotically null controllable with bounded controls (ANCBC). For ANCBC systems subject to actuator saturation, various feedback laws that achieve global or semi-global stabilization on the null controllable region have been constructed (see, for example, [19], [20], and [24]-[26]). Here by semiglobal stabilization on the null controllable region we mean the construction of a feedback law that achieves a domain of attraction large enough to include any a priori given (arbitrarily large) bounded set in the null controllable region.

For exponentially unstable open-loop systems subject to actuator saturation, it was shown in [27] that the problem of stabilization can be reduced to one of stabilizing its antistable subsystem, whose null controllable region is a bounded convex open set. A complete characterization of the null controllable region for a general linear system was developed in [10], and simple feedback laws were constructed that achieve semiglobal stabilization on the null controllable region for linear systems with only two exponentially unstable poles in [11]. In [12], feedback laws were constructed to achieve semiglobal stabilization on the null controllable region for general linear systems subject to actuator saturation.

In comparison with the problem of stabilization, the problem of output regulation for linear systems subject to control constraint, however, has received relatively less attention. The few works that have motivated our current research are [1], [2], [21], [26]. In [21] and [26], the problem of output regulation was studied for ANCBC systems subject to actuator saturation. In [21], necessary and sufficient conditions on the plant/exosystem and their initial conditions were derived under which output regulation can be achieved, and feedback laws that achieve semiglobal output regulation were constructed. In [1] and [2], the authors made an attempt to address the problem of output regulation for exponentially unstable linear systems subject to actuator saturation. The attempt was to enlarge the set of initial conditions of the plant and the exosystem under which output regulation can be achieved. In particular, for plants with only one positive pole and exosystems that contain only one frequency component, feedback laws were constructed that achieve output regulation on what we will characterize in this paper as the regulatable region.

The objective of this paper is to systematically study the problem of output regulation for general linear systems with bounded controls. Unlike in the case for a linear system without control constraint, where output regulation can be achieved for all initial states, here in the presence of control constraint, the set of initial states for which output regulation is possible may not be the whole state-space. For instance, suppose that we have an exponentially unstable plant, then it is known that 
the set of initial states of the plant that can be kept bounded with constrained controls is not the whole state-space. The set of initial states of the plant where output regulation is possible is even more restricted. For this reason, we will start our investigation by characterizing the set of initial states of the plant and the exosystem where output regulation is possible and we will call this set the regulatable region. It turns out that the regulatable region can be characterized in terms of the null controllable region of the antistable subsystem of the plant.

We then proceed to construct output regulation laws from a given stabilizing state feedback law. We show that a stabilizing feedback law that achieves a larger domain of attraction leads to a feedback law that achieves output regulation on a larger subset of the regulatable region and, a stabilizing feedback law on the entire null controllable region leads to a feedback law that achieves output regulation on the entire regulatable region.

This paper generalizes and enhances our earlier results in [7]. First, in [7], we restricted our attention to systems whose actuators are subject to symmetric saturation. In practical systems, the actuators may subject to asymmetric saturation and there may exist coupling among different actuators. For this reason, we consider general convex control constraint in this paper. Second, the controllers constructed in [7] have a switching nature and are discontinuous at the switching surfaces. In this paper, we will construct continuous feedback laws for output regulation.

The remainder of this paper is organized as follows. In Section II, we state the problem of output regulation for linear systems with bounded controls. Section III characterizes the regulatable region. Sections IV and V respectively construct state feedback and error feedback laws that achieve output regulation on the regulatable region. Section VI includes a numerical example along with some discussion on robustness issues. Finally, Section VII draws a brief conclusion to our current work.

Throughout this paper, we will use standard notation. For a vector $u \in \mathbf{R}^{m}$, we use $|u|_{\infty}$ and $|u|_{2}$ to denote the vector $\infty$-norm and the two-norm. For a measurable function $u:[0, \infty) \rightarrow \mathbf{R}^{m}$, we define $\|u\|_{\infty}=\sup _{t \in[0, \infty]}|u(t)|_{\infty}$. We use $\operatorname{sat}(\cdot)$ to denote the standard saturation function, i.e., the $i$ th component of $\operatorname{sat}(u)$ is $\operatorname{sgn}\left(u_{i}\right) \min \left\{1,\left|u_{i}\right|\right\}$.

\section{PRELIMINARIES AND PROBlem Statement}

In this section, we first recall from [4] and [16] the classical formulation and results on the problem of output regulation for linear systems. This brief review will motivate our formulation as well as the solution to the problem of output regulation for linear systems with bounded controls.

Consider a linear system

$$
\begin{aligned}
\dot{x} & =A x+B u+P w \\
\dot{w} & =S w \\
e & =C x+Q w .
\end{aligned}
$$

The first equation of this system describes a plant, with state $x \in \mathbf{R}^{n}$ and input $u \in \mathbf{R}^{m}$, subject to the effect of a disturbance represented by $P w$. The third equation defines the error $e \in \mathbf{R}^{q}$ between the actual plant output $C x$ and a reference signal $-Q w$ that the plant output is required to track. The second equation describes an autonomous system, often called the exosystem, with state $w \in \mathbf{R}^{r}$. The exosystem models the class of disturbances and references taken into consideration.

The control action to the plant, $u$, can be provided either by state feedback or by error feedback. The objective is to achieve internal stability and output regulation. Internal stability means that if we disconnect the exosystem and set $w$ equal to zero then the closed-loop system is asymptotically stable. Output regulation means that, for any initial conditions of the plant and the exosystem, the state of the plant is bounded and $e(t) \rightarrow 0$ as $t \rightarrow \infty$.

The solution to the output regulation problem was first obtained by Francis in [4]. It is now well known that under some mild necessary assumptions, the output regulation problem is solvable if and only if there exist matrices $\Pi$ and $\Gamma$ that solve the linear matrix equations

$$
\begin{aligned}
\Pi S & =A \Pi+B \Gamma+P, \\
C \Pi+Q & =0 .
\end{aligned}
$$

For more details about the assumptions and the solution, see [4] and [16].

In this paper, we study the problem of output regulation for the linear system (1) subject to control constraint. The control constraint is described by a compact convex set $\mathcal{U} \subset \mathbf{R}^{m}$ that contains the origin in its interior. A control $u(\cdot)$ is said to be admissible if $u(\cdot)$ is measurable and $u(t) \in \mathcal{U}$ for all $t \in \mathbf{R}$. Denote

$$
\alpha \mathcal{U}=\{\alpha u: u \in \mathcal{U}\}
$$

Given $\delta \in[0,1], u_{1} \in \delta \mathcal{U}$ and $u_{2} \in(1-\delta) \mathcal{U}$, it is easy to see that $u_{1}+u_{2} \in \mathcal{U}$ by the convexity of $\mathcal{U}$.

Following [4], [16] and [21], we make the following necessary assumptions on the plant and the exosystem.

A1) The matrix (2) has solution $(\Pi, \Gamma)$;

A2) The matrix $S$ has all its eigenvalues on the imaginary axis and is neutrally stable.

A3) The pair $(A, B)$ is stabilizable.

A4) The initial state $w_{0}$ of the exosystem is in the following set:

$$
\mathcal{W}_{0}=\left\{w_{0} \in \mathbf{R}^{r}: \Gamma e^{S t} w_{0} \in \rho \mathcal{U}, \forall t \geq 0\right\}
$$

for some $\rho \in[0,1)$ and $\mathcal{W}_{0}$ is compact. For later use, we denote $\delta=1-\rho$.

We note that the compactness of $\mathcal{W}_{0}$ can be guaranteed by the observability of $(\Gamma, S)$. Indeed, if $(\Gamma, S)$ is not observable, then the exosystem can be reduced to make it so. As will be seen shortly, the exosystem affects the output regulation property through the signal $\Gamma w(t)$. It is also clear that if $w_{0} \in \mathcal{W}_{0}$, then $w(t)=e^{S t} w_{0} \in \mathcal{W}_{0}$, for all $t \geq 0$.

Under the control constraint, some initial conditions of the plant and exosystem may make it impossible to keep the state bounded or to drive the tracking error to 0 asymptotically. For instance, if the matrix $A$ has some eigenvalues in the right-half plane, there always exist some initial states which will make the state trajectory go unbounded no matter what admissible control is applied. In view of this, the first task of this paper is to characterize the set of initial conditions for which there exist 
admissible controls to keep the state bounded and to drive the tracking error to 0 asymptotically.

\section{REgULATABLE REGION}

To begin with, we define a new state $z=x-\Pi w$ and rewrite the system equations as

$$
\begin{aligned}
\dot{z} & =A z+B u-B \Gamma w \\
\dot{w} & =S w \\
e & =C z .
\end{aligned}
$$

This particular state transformation has been traditionally used in the output regulation literature to transform the output regulation problem into a stabilization problem. The remaining part of this paper will be focused on system (4). All the results can be easily restated in terms of the original state of the plant $x$ by replacing $z$ with $x-\Pi w$. Here we note that, when $w=0$, the internal stability in terms of $z$ is the same as that in terms of $x$. As to output regulation, it is clear that $e(t)$ goes to zero asymptotically if $z(t)$ does. To combine the objectives of achieving internal stability and achieving output regulation, we will define the notion of regulatable region in terms of driving $z(t)$ to zero instead of driving $e(t)$ to zero. As will be explained in detail in Remark 1, this will result in essentially the same description of the regulatable region and will avoid some tedious technical discussions.

\section{Definition 1:}

1) Given a $T>0$, a pair $\left(z_{0}, w_{0}\right) \in \mathbf{R}^{n} \times \mathbf{R}^{r}$ is regulatable in time $T$ if there exists an admissible control $u(\cdot)$, such that the response of (4) satisfies $z(t)=0$, for all $t \geq T$.

2) A pair $\left(z_{0}, w_{0}\right)$ is regulatable if there exist a finite $T>0$ and an admissible control $u(\cdot)$ such that $z(t)=0$, for all $t \geq T$.

3) The set of all $\left(z_{0}, w_{0}\right)$ regulatable in time $T$ is denoted as $\mathcal{R}_{g}(T)$ and the set of all regulatable $\left(z_{0}, w_{0}\right)$ is referred to as the regulatable region and is denoted as $\mathcal{R}_{g}$.

4) The set of all $\left(z_{0}, w_{0}\right)$ for which there exists an admissible control $u(\cdot)$ such that the response of (4) satisfies $\lim _{t \rightarrow \infty} z(t)=0$ is referred to as the asymptotically regulatable region and is denoted as $\mathcal{R}_{g}^{a}$.

It is clear that, if $\left(z_{0}, w_{0}\right) \in \mathcal{R}_{g}$, we must have $\Gamma e^{S t} w_{0} \in \mathcal{U}$ for all $t \geq 0$, since the only way to keep $z(t)=0$ for all $t>T$ is to use a control $u \in \mathcal{U}$ to cancel the term $\Gamma w$ for all $t>T$. This justifies Assumption A4). Because of Assumption A4), the requirement in Definition 1 that $z(t)=0$, for all $t \geq T$ can be replaced with $z(T)=0$ and it is clear that $\mathcal{R}_{g}\left(T_{1}\right) \subset \mathcal{R}_{g}\left(T_{2}\right)$ if $T_{2}>T_{1}$.

We will describe $\mathcal{R}_{g}(T), \mathcal{R}_{g}$ and $\mathcal{R}_{g}^{a}$ in terms of the null controllable region of the plant $\dot{v}=A v+B u, u(t) \in \mathcal{U}$, which is defined as follows.

Definition 2: The null controllable region in time $T$, denoted as $\mathcal{C}(T)$, is the set of $v_{0} \in \mathbf{R}^{n}$ that can be driven to the origin in time $T$ by admissible controls. The null controllable region, denoted as $\mathcal{C}$, is the set of $v_{0} \in \mathbf{R}^{n}$ that can be driven to the origin in a finite time by admissible controls. The asymptotically null controllable region, denoted as $\mathcal{C}^{a}$, is the set of all $v_{0}$ that can be driven to the origin asymptotically by admissible controls.
Clearly, $\mathcal{C}=\bigcup_{T \in[0, \infty)} \mathcal{C}(T)$ and

$$
\begin{aligned}
& \mathcal{C}(T) \\
& \quad=\left\{v_{0} \in \mathbf{R}^{n}: v_{0}=-\int_{0}^{T} e^{-A \tau} B u(\tau) d \tau, u(\tau) \in \mathcal{U} \forall \tau\right\} .
\end{aligned}
$$

Here, we note that the minus sign "-" before the integral can be removed only if $\mathcal{U}$ is symmetric. It is also clear that the null controllable region and the asymptotically null controllable region are identical if the pair $(A, B)$ is controllable. Some simple methods to describe $\mathcal{C}$ and $\mathcal{C}^{a}$ were recently developed in [10] for the case where $\mathcal{U}$ is a unit box.

To simplify the characterization of $\mathcal{R}_{g}$ and $\mathcal{R}_{g}^{a}$, let us assume, without loss of generality, that $z=\left[\begin{array}{l}z_{1} \\ z_{2}\end{array}\right], z_{1} \in \mathbf{R}^{n_{1}}, z_{2} \in \mathbf{R}^{n_{2}}$, and

$$
A=\left[\begin{array}{cc}
A_{1} & 0 \\
0 & A_{2}
\end{array}\right] \quad B=\left[\begin{array}{l}
B_{1} \\
B_{2}
\end{array}\right]
$$

where $A_{1} \in \mathbf{R}^{n_{1} \times n_{1}}$ is semistable (i.e., all its eigenvalues are in the closed left-half plane) and $A_{2} \in \mathbf{R}^{n_{2} \times n_{2}}$ is antistable (i.e., all its eigenvalues are in the open right-half plane). The system including the antistable subplant and the exosystem

$$
\begin{aligned}
\dot{z}_{2} & =A_{2} z_{2}+B_{2} u-B_{2} \Gamma w \\
\dot{w} & =S w
\end{aligned}
$$

is of crucial importance. Denote its regulatable regions as $\mathcal{R}_{g_{2}}(T), \mathcal{R}_{g_{2}}$ and $\mathcal{R}_{g_{2}}{ }^{a}$, and the null controllable regions for the system $\dot{v}_{2}=A_{2} v_{2}+B_{2} u$ as $\mathcal{C}_{2}(T)$ and $\mathcal{C}_{2}$. Then, the asymptotically null controllable region for the system $\dot{v}=A v+B u$ is given by $\mathcal{C}^{a}=\mathbf{R}^{n_{1}} \times \mathcal{C}_{2}$ (see, e.g., [6]), where $\mathcal{C}_{2}$ is a bounded convex open set. Denote the closure of $\mathcal{C}_{2}$ as $\overline{\mathcal{C}_{2}}$, then

$\overline{\mathcal{C}}_{2}$

$$
=\left\{v_{20} \in \mathbf{R}^{n_{2}}: v_{20}=-\int_{0}^{\infty} e^{-A_{2} \tau} B_{2} u(\tau) d \tau, u(\tau) \in \mathcal{U} \forall \tau\right\} .
$$

Also, if $D$ is a closed subset of $\mathcal{C}_{2}$, then there is a finite $T>0$ such that $D \subset \mathcal{C}_{2}(T)$.

Theorem 1: Let $V_{2} \in \mathbf{R}^{n_{2} \times r}$ be the unique solution to the matrix equation

$$
-A_{2} V_{2}+V_{2} S=-B_{2} \Gamma
$$

and let $V(T)=V_{2}-e^{-A_{2} T} V_{2} e^{S T}$. Then, the following hold.

1) $\mathcal{R}_{g_{2}}(T)=\left\{\left(z_{20}, w_{0}\right) \in \mathbf{R}^{n_{2}} \times \mathcal{W}_{0}: z_{20}-V(T) w_{0} \in \mathcal{C}_{2}(T)\right\}$

2) $\mathcal{R}_{g_{2}}=\left\{\left(z_{20}, w_{0}\right) \in \mathbf{R}^{n_{2}} \times \mathcal{W}_{0}: z_{20}-V_{2} w_{0} \in \mathcal{C}_{2}\right\}$.

3) $\mathcal{R}_{g}^{a}=R^{n_{1}} \times \mathcal{R}_{g_{2}}$.

4) If $z_{20}-V_{2} w_{0} \notin \overline{\mathcal{C}_{2}}$, then $z_{2}(t)$ will grow unbounded whatever admissible control is applied.

Proof:

1) Given $\left(z_{20}, w_{0}\right) \in \mathbf{R}^{n_{2}} \times \mathcal{W}_{0}$ and an admissible control $u$, the solution of (7) at $t=T$ is

$$
\begin{aligned}
& z_{2}(T)=e^{A_{2} T} \\
& \times\left(z_{20}+\int_{0}^{T} e^{-A_{2} \tau} B_{2} u(\tau) d \tau-\int_{0}^{T} e^{-A_{2} \tau} B_{2} \Gamma e^{S \tau} w_{0} d \tau\right) .
\end{aligned}
$$


Since $-A_{2} V_{2}+V_{2} S=-B_{2} \Gamma$, we have

$$
\begin{aligned}
\frac{d e^{-A_{2} \tau} V_{2} e^{S \tau}}{d \tau} & =e^{-A_{2} \tau}\left(-A_{2} V_{2}\right) e^{S \tau}+e^{-A_{2} \tau} V_{2} S e^{S \tau} \\
& =e^{-A_{2} \tau}\left(-A_{2} V_{2}+V_{2} S\right) e^{S \tau} \\
& =-e^{-A_{2} \tau} B_{2} \Gamma e^{S \tau}
\end{aligned}
$$

noting that $A_{2}$ and $e^{-A_{2} \tau}$ commute, and $S$ and $e^{S \tau}$ commute. Hence

$$
\begin{aligned}
-\int_{0}^{T} e^{-A_{2} \tau} B_{2} \Gamma e^{S \tau} d \tau & =\int_{0}^{T} d e^{-A_{2} \tau} V_{2} e^{S \tau} \\
& =e^{-A_{2} T} V_{2} e^{S T}-V_{2}=-V(T) .
\end{aligned}
$$

Therefore

$e^{-A_{2} T} z_{2}(T)=z_{20}-V(T) w_{0}+\int_{0}^{T} e^{-A_{2} \tau} B_{2} u(\tau) d \tau$

To prove 1), it suffices to show that $z_{20}-V(T) w_{0} \in$ $\mathcal{C}_{2}(T) \Longleftrightarrow\left(z_{20}, w_{0}\right) \in \mathcal{R}_{g_{2}}(T)$.

If $z_{20}-V(T) w_{0} \in \mathcal{C}_{2}(T)$, then by (5), there exists an admissible control $u_{1}$ such that

$$
z_{20}-V(T) w_{0}=-\int_{0}^{T} e^{-A_{2} \tau} B_{2} u_{1}(\tau) d \tau
$$

Let $u(t)=u_{1}(t)$ for $t \in[0, T]$ and $u(t)=\Gamma w(t)$ for $t>T$, then $u$ is admissible by Assumption A4, and it follows from (11) that $z_{2}(T)=0$ and $z_{2}(t)=0$ for all $t>$ $T$. Therefore, $\left(z_{20}, w_{0}\right) \in \mathcal{R}_{g_{2}}(T)$. On the other hand, if $\left(z_{20}, w_{0}\right) \in \mathcal{R}_{g_{2}}(T)$, then there exists an admissible $u$ such that $z_{2}(T)=0$. Also by (11), we have

$$
z_{20}-V(T) w_{0}+\int_{0}^{T} e^{-A_{2} \tau} B_{2} u(\tau) d \tau=0
$$

which implies that $z_{20}-V(T) w_{0} \in \mathcal{C}_{2}(T)$.

2) Since $A_{2}$ is antistable and $S$ is stable, we have that $\lim _{T \rightarrow \infty} V(T)=V_{2}$. It follows from (10) that

$$
V_{2}=\int_{0}^{\infty} e^{-A_{2} \tau} B_{2} \Gamma e^{S \tau} d \tau
$$

First, we show that $z_{20}-V_{2} w_{0} \in \mathcal{C}_{2} \Longrightarrow\left(z_{20}, w_{0}\right) \in$ $\mathcal{R}_{g_{2}}$. Since $\mathcal{C}_{2}$ is open, there exists an $\varepsilon>0$ such that $\left\{z_{20}-V_{2} w_{0}+z_{2}:\left|z_{2}\right|_{\infty} \leq \varepsilon\right\} \subset \mathcal{C}_{2}$. Also, there exists a $T_{1}>0$ such that $\left\{z_{20}-V_{2} w_{0}+z_{2}:\left|z_{2}\right|_{\infty} \leq \varepsilon\right\} \subset$ $\mathcal{C}_{2}\left(T_{1}\right)$. Since $\lim _{T \rightarrow \infty} V(T)=V_{2}$, there is a $T_{2}>T_{1}$ such that $z_{20}-V\left(T_{2}\right) w_{0} \in \mathcal{C}_{2}\left(T_{1}\right) \subset \mathcal{C}_{2}\left(T_{2}\right)$. It follows from 1) that $\left(z_{20}, w_{0}\right) \in \mathcal{R}_{g_{2}}\left(T_{2}\right) \subset \mathcal{R}_{g_{2}}$.

Next, we show that $\left(z_{20}, w_{0}\right) \in \mathcal{R}_{g_{2}} \quad \Longrightarrow$ $z_{20}-V_{2} w_{0} \in \mathcal{C}_{2}$. If $\left(z_{20}, w_{0}\right) \in \mathcal{R}_{g_{2}}$, then $\left(z_{20}, w_{0}\right) \in \mathcal{R}_{g_{2}}\left(T_{1}\right)$ for some $T_{1}>0$. It follows from the definition of $\mathcal{R}_{g}(T)$ that there is an admissible control $u_{1}$ such that

$z_{20}-\int_{0}^{T_{1}} e^{-A_{2} \tau} B_{2} \Gamma e^{S \tau} w_{0} d \tau+\int_{0}^{T_{1}} e^{-A_{2} \tau} B_{2} u_{1}(\tau) d \tau=0$.
Denote $Z_{2}=\left\{\delta e^{-A_{2} T_{1}} v_{2}: v_{2} \in \overline{\mathcal{C}}_{2}\right\}$. For each $z_{2} \in$ $Z_{2}$, there is an admissible control $u_{2}$ such that

$$
\begin{aligned}
z_{2} & =-\delta e^{-A_{2} T_{1}} \int_{0}^{\infty} e^{-A_{2} \tau} B_{2} u_{2}(\tau) d \tau \\
& =-\int_{T_{1}}^{\infty} e^{-A_{2} \tau} B_{2} \delta u_{2}\left(\tau-T_{1}\right) d \tau .
\end{aligned}
$$

It follows from (12) and (13) that

$$
\begin{aligned}
z_{20}- & V_{2} w_{0}+z_{2} \\
= & z_{20}-\int_{0}^{\infty} e^{-A_{2} \tau} B_{2} \Gamma e^{S \tau} w_{0} d \tau \\
& \quad-\int_{T_{1}}^{\infty} e^{-A_{2} \tau} B_{2} \delta u_{2}\left(\tau-T_{1}\right) d \tau \\
= & z_{20}-\int_{0}^{T_{1}} e^{-A_{2} \tau} B_{2} \Gamma e^{S \tau} w_{0} d \tau \\
& \quad-\int_{T_{1}}^{\infty} e^{-A_{2} \tau} B_{2}\left(\delta u_{2}\left(\tau-T_{1}\right)+\Gamma e^{S \tau} w_{0}\right) d \tau \\
= & -\int_{0}^{T_{1}} e^{-A_{2} \tau} B_{2} u_{1}(\tau) d \tau \\
& -\int_{T_{1}}^{\infty} e^{-A_{2} \tau} B_{2}\left(\delta u_{2}\left(\tau-T_{1}\right)+\Gamma e^{S \tau} w_{0}\right) d \tau \in \overline{\mathcal{C}}_{2} .
\end{aligned}
$$

The last step follows from the fact that $\Gamma e^{S \tau} w_{0} \in(1-\delta) \mathcal{U}$ and $\delta u_{2}\left(\tau-T_{1}\right) \in \delta \mathcal{U}$ for all $\tau \geq T_{1}$, and that the input $u(t)=u_{1}(t)$ for $t \in\left[0, T_{1}\right], u(t)=\delta u_{2}\left(t-T_{1}\right)+\Gamma e^{S t} w_{0}$ for $t>T_{1}$ is admissible. This implies that

$$
\left\{z_{20}-V_{2} w_{0}+z_{2}: z_{2} \in Z_{2}\right\} \subset \overline{\mathcal{C}}_{2} .
$$

Since $e^{-A_{2} T_{1}}$ is nonsingular, the set $Z_{2}$ contains the origin in its interior. It follows that $z_{20}-V_{2} w_{0} \in \mathcal{C}_{2}$.

3) We first show that $\mathcal{R}_{g_{2}}^{a}=\mathcal{R}_{g_{2}}$. It is easy to see that $\mathcal{R}_{g_{2}} \subset \mathcal{R}_{g_{2}}^{a}$. To show $\mathcal{R}_{g_{2}}^{a} \subset \mathcal{R}_{g_{2}}$, suppose we are given $z_{20} \in \mathcal{R}_{g_{2}}^{a}$. Then there exits a finite time $T$ and an admissible control such that $z_{2}(T) \in \delta \mathcal{C}_{2}$ since $\delta \mathcal{C}_{2}$ is an open set containing the origin. For $t>T$, let $u(t)=$ $\Gamma w(t)+u_{\delta}(t)$ with $u_{\delta}(t) \in \delta \mathcal{U}$. Since $w_{0} \in \mathcal{W}_{0}$, we have $\Gamma w(t) \in(1-\delta) \mathcal{U}$ and, hence, $u(t) \in \mathcal{U}$ and

$$
\dot{z}_{2}=A_{2} z_{2}+B_{2} u-B_{2} \Gamma w=A_{2} z_{2}+B_{2} u_{\delta} .
$$

Since $z_{2}(T) \in \delta \mathcal{C}_{2}$, we have a control $u_{\delta}$ and a finite $T_{1}>T$ such that $z_{2}\left(T_{1}\right)=0$. So, we have $z_{20} \in \mathcal{R}_{g_{2}}$, and hence $\mathcal{R}_{g_{2}}{ }^{a} \subset \mathcal{R}_{g_{2}}$. Therefore, $\mathcal{R}_{g_{2}}{ }^{a}=\mathcal{R}_{g_{2}}$.

Now, we show that $\mathcal{R}_{g}^{a} \subset \mathbf{R}^{n_{1}} \times \mathcal{R}_{g_{2}}$. Suppose $\left(z_{0}, w_{0}\right)=\left(z_{10}, z_{20}, w_{0}\right) \in \mathcal{R}_{g}^{a}$, then there exists an admissible control such that $\lim _{t \rightarrow \infty} z_{2}(t)=0$. This implies that $\left(z_{20}, w_{0}\right) \subset \mathcal{R}_{g_{2}}^{a}=\mathcal{R}_{g_{2}}$.

We next show that $\mathbf{R}^{n_{1}} \times \mathcal{R}_{g_{2}} \subset \mathcal{R}_{g}^{a}$. Suppose $\left(z_{10}, z_{20}, w_{0}\right) \in \mathbf{R}^{n_{1}} \times \mathcal{R}_{g_{2}}$, then there exist a $T \geq 0$ and an admissible control such that $z_{2}(T)=0$. Since $\left[\begin{array}{c}z_{1}(T) \\ 0\end{array}\right]$ is inside the asymptotically null controllable region for the system $\dot{z}=A z+B u_{\delta}$ under the constraint $u_{\delta}(t) \in \delta \mathcal{U}$ (by [6]), there exists a $u=\Gamma w+u_{\delta}$ for $t>T$ such that $\lim _{t \rightarrow \infty} z(t)=0$. Hence $\left(z_{0}, w_{0}\right) \in \mathcal{R}_{g}^{a}$. 
This establishes that $\mathbf{R}^{n_{1}} \times \mathcal{R}_{g_{2}} \subset \mathcal{R}_{g}^{a}$ and hence $\mathbf{R}^{n_{1}} \times \mathcal{R}_{g_{2}}=\mathcal{R}_{g}^{a}$.

4) Suppose that $z_{20}-V_{2} w_{0} \notin \overline{\mathcal{C}_{2}}$, then there exists an $\varepsilon>0$ such that $\left|z_{20}-V_{2} w_{0}-z_{2}\right|_{2}>\varepsilon \forall z_{2} \in \overline{\mathcal{C}}_{2}$. Since

$$
\begin{gathered}
\lim _{t \rightarrow \infty} \int_{0}^{t} e^{-A_{2} \tau} B_{2} \Gamma e^{S \tau} w_{0} d \tau=V_{2} w_{0}, \\
\text { and }-\int_{0}^{t} e^{-A_{2} \tau} B_{2} u(\tau) d \tau \in \mathcal{C}_{2} \quad \forall t>0, u(\tau) \in \mathcal{U} \\
\text { there exists a } t_{0}>0 \text { such that } \\
\left|z_{20}-\int_{0}^{t} e^{-A_{2} \tau} B_{2} \Gamma e^{S \tau} w_{0} d \tau+\int_{0}^{t} e^{-A_{2} \tau} B_{2} u(\tau) d \tau\right|_{2} \\
>\frac{\varepsilon}{2} \quad \forall t>t_{0} .
\end{gathered}
$$

Since the smallest singular value of $e^{A_{2} t}$ increases exponentially with $t$, it follows from (9) that $z_{2}(t)$ will grow unbounded whatever admissible control is applied.

Remark 1: Here, we justify the requirement of driving $z(t)$, instead of $e(t)$, to zero in Definition 1. From the previous theorem, we see that $\left(z_{0}, w_{0}\right)$ is regulatable if and only if $z_{20}-$ $V_{2} w_{0} \in \mathcal{C}_{2}$. By item 4 ) of the theorem, this is essentially the necessary condition to keep $z_{2}(t)$ bounded. Hence, even if the requirement $\lim _{t \rightarrow \infty} z(t)=0$ is replaced with $\lim _{t \rightarrow \infty} e(t)=0$, we still require $z_{20}-V_{2} w_{0} \in \overline{\mathcal{C}}_{2}$ to achieve output regulation. The gap only arises from the boundary of $\mathcal{C}_{2}$. It is unclear whether it is possible to achieve $\lim _{t \rightarrow \infty} e(t)=0$ with $z(t)$ bounded for $z_{20}-V_{2} w_{0} \in \partial \mathcal{C}_{2}$. Since this problem involves too much technical detail and is of little practical importance (we will not take the risk to allow $z_{20}-V_{2} w_{0} \in \partial \mathcal{C}_{2}$, otherwise a small perturbation may cause the state to grow unbounded), we will not address this subtle technical point here.

Remark 2: We now interpret the characterization of the regulatable region in terms of the original state $x$. Without loss of generality, we also assume that $A$ and $B$ are partitioned as in (6). Suppose that $x, \Pi$ and $P$ are partitioned accordingly as

$$
x=\left[\begin{array}{l}
x_{1} \\
x_{2}
\end{array}\right] \quad \Pi=\left[\begin{array}{l}
\Pi_{1} \\
\Pi_{2}
\end{array}\right] \quad P=\left[\begin{array}{l}
P_{1} \\
P_{2}
\end{array}\right] .
$$

Then $z_{2}=x_{2}-\Pi_{2} w$. Hence $\left(z_{0}, w_{0}\right) \in \mathcal{R}_{g}^{a}$ if and only if $x_{20}-\left(\Pi_{2}+V_{2}\right) w_{0} \in \mathcal{C}_{2}$. Combining (2) and (8), it is easy to verify that

$$
\left(\Pi_{2}+V_{2}\right) S=A_{2}\left(\Pi_{2}+V_{2}\right)+P_{2} .
$$

Therefore, if we redefine the regulatable region in terms of $x$, it would be the set of $\left(x_{0}, w_{0}\right)$ such that $x_{20}-\tilde{V}_{2} w_{0} \in \mathcal{C}_{2}$, where $\tilde{V}_{2}$ satisfies

$$
\tilde{V}_{2} S-A_{2} \tilde{V}_{2}=P_{2}
$$

\section{State FeEdBack Controller}

In view of what has been discussed in the previous section, the set of initial conditions for which output regulation can be achieved with any feedback law must be a subset of $\mathcal{R}_{g}^{a}$. In this section, we would like to search for a state feedback law such that this subset is as large as possible, or, as close as possible to $\mathcal{R}_{g}^{a}$.

Consider the connection of the system

$$
\begin{aligned}
\dot{z} & =A z+B u-B \Gamma w \\
\dot{w} & =S w
\end{aligned}
$$

with a dynamic feedback

$$
\begin{array}{ll}
u=g(\alpha, z, w) & g(\alpha, z, w) \in \mathcal{U} \\
\dot{\alpha}=h(\alpha, z, w) & \alpha(0)=\alpha_{0}
\end{array}
$$

where $\alpha=\left[\begin{array}{c}\alpha_{1} \\ \alpha_{2}\end{array}\right] \in \mathbf{R}^{2}$ and $\alpha_{0}$ is the initial value of $\alpha$, which will be a fixed vector. Denote the time response of $z(t)$ to the initial state $\left(z_{0}, w_{0}\right)$ as $z\left(t, z_{0}, w_{0}\right)$ and define

$$
\mathcal{S}_{z w}:=\left\{\left(z_{0}, w_{0}\right) \in \mathbf{R}^{n} \times \mathcal{W}_{0}: \lim _{t \rightarrow \infty} z\left(t, z_{0}, w_{0}\right)=0\right\} .
$$

Then, $\mathcal{S}_{z w}$ is the set of initial conditions where output regulation is achieved by (16). It is clear that $\mathcal{S}_{z w} \subset \mathcal{R}_{g}^{a}$. Our objective is to design a control law of the form (16) such that $\mathcal{S}_{z w}$ is as large as possible, or as close to $\mathcal{R}_{g}^{a}$ as possible.

We assume that a continuous stabilizing state feedback law $u=f(v)$, where $f: \mathbf{R}^{n} \rightarrow \mathcal{U}$, has been designed and the equilibrium $v=0$ of the closed-loop system

$$
\dot{v}=A v+B f(v)
$$

has a domain of attraction $\mathcal{S} \subset \mathcal{C}^{a}$. In addition, we also assume that there exists a positive number $d_{0}>0$ such that the system

$$
\dot{v}=A v+B f(v)+\eta \quad|\eta|_{\infty} \leq d_{0}
$$

has a bounded invariant set $\mathcal{S}_{I} \subset \mathcal{S}$ which contains the origin in its interior, and

$$
\lim _{t \rightarrow \infty} v(t)=0
$$

as long as $\lim _{t \rightarrow \infty} \eta(t)=0$ and $v(0) \in \mathcal{S}_{I}$. The invariance of $\mathcal{S}_{I}$ in the presence of disturbance $\eta$ is imposed to allow the dynamics of $\alpha$, which will be made clear shortly. This is crucial for the continuity of the control $u$ in (16), as compared with the switching feedback law in [7]. In [11], we designed a feedback law of the form $u=\operatorname{sat}(F v)$ for systems with two antistable poles to achieve semiglobal stabilization for systems subject to input saturation. This means that $\mathcal{S}$ can be made to include any bounded subset of $\mathcal{C}^{a}$. Since this feedback law is locally linear, there always exists a bounded invariant set $\mathcal{S}_{I}$, in particular, an invariant ellipsoid, over which the desired disturbance rejection property is ensured. For more general systems, an LMI optimization method was proposed in [9] for designing feedback laws to achieve the desired disturbance rejection property. In [9], the domain of attraction $\mathcal{S}$ was estimated with an invariant ellipsoid and the objective was to maximize the invariant ellipsoid.

We also make the assumption that there exists a matrix $V \in$ $\mathbf{R}^{n \times r}$ such that

$$
-A V+V S=-B \Gamma .
$$


This will be the case if $A$ and $S$ have no common eigenvalues. With the decomposition in (6), if we partition $V$ accordingly as $\left[\begin{array}{l}V_{1} \\ V_{2}\end{array}\right]$, then $V_{2}$ satisfies $-A_{2} V_{2}+V_{2} S=-B_{2} \Gamma$.

Denote

$$
D_{z w}:=\left\{(z, w) \in \mathbf{R}^{n} \times \mathcal{W}_{0}: z-V w \in \mathcal{S}\right\} .
$$

From Theorem 1 and $\mathcal{C}^{a}=\mathbf{R}^{n_{1}} \times \mathcal{C}_{2}$, we see that

a) the set $D_{z w}$ increases as $\mathcal{S}$ increases, and if $\mathcal{S}=\mathcal{C}^{a}$, then $D_{z w}=\mathcal{R}_{g}^{a}$

b) in the absence of $w, x_{0} \in \mathcal{S} \Rightarrow\left(z_{0}, 0\right) \in D_{z w}$.

We will construct an output regulation law (16) from the stabilizing feedback law $u=f(v)$ in such a way that $D_{z w} \subset \mathcal{S}_{z w}$. It is then clear that larger $\mathcal{S}$ will lead to larger $\mathcal{S}_{z w}$. Moreover, if $f$ is chosen such that $\mathcal{S}=\mathcal{C}^{a}$, then $D_{z w}=\mathcal{R}_{g}^{a}$ and $\mathcal{S}_{z w}=\mathcal{R}_{g}^{a}$. In view of these arguments, our objective of producing a large $\mathcal{S}_{z w}$ can be achieved by designing $f$ such that $\mathcal{S}$ is as large as possible and to construct an output regulation law (16) from $f$ such that $D_{z w} \subset \mathcal{S}_{z w}$. Efforts have been made to enlarge $\mathcal{S}$ in [9] and [11]. Here, we focus on the construction of an output regulation law (16) from a given $f$ such that $D_{z w} \subset \mathcal{S}_{z w}$.

First, let us consider a simple feedback law $u=f(z-V w)$ and see what it can achieve for $\left(z_{0}, w_{0}\right) \in D_{z w}$.

Lemma 1: For system (15), let $u=f(z-V w)$. Consider the closed-loop system

$$
\begin{aligned}
\dot{z} & =A z+B f(z-V w)-B \Gamma w \\
\dot{w} & =S w .
\end{aligned}
$$

For this system, $D_{z w}$ is an invariant set and for all $\left(z_{0}, w_{0}\right) \in$ $D_{z w}, \lim _{t \rightarrow \infty}(z(t)-V w(t))=0$.

Proof: Because of (19), we can replace $-B \Gamma$ in (21) with $-A V+V S$, i.e.,

$$
\begin{aligned}
\dot{z} & =A z+B f(z-V w)-A V w+V S w \\
& =A(z-V w)+B f(z-V w)+V \dot{w} .
\end{aligned}
$$

Define the new state $v:=z-V w$, we have

$$
\dot{v}=A v+B f(v)
$$

which has a domain of attraction $\mathcal{S}$. This also implies that $\mathcal{S}$ is an invariant set for the $v$-system.

If $\left(z_{0}, w_{0}\right) \in D_{z w}$, then $v_{0}=z_{0}-V w_{0} \in \mathcal{S}$. It follows that $v(t)=z(t)-V w(t) \in \mathcal{S}$ for all $t \geq 0$, which means that $D_{z w}$ is invariant, and $\lim _{t \rightarrow \infty}(z(t)-V w(t))=\lim _{t \rightarrow \infty} v(t)=0$.

Lemma 1 says that, for any $\left(z_{0}, w_{0}\right) \in D_{z w}$, the feedback $u=f(z-V w)$ will cause $z(t)-V w(t)$ to approach zero and $z(t)$ to approach $V w(t)$, which is bounded. In [7], a finite sequence of control laws were constructed to cause $z(t)$ to approach $\alpha^{k} V w(t)$ with a constant $\alpha \in(0,1)$ and increasing integers $k>0$. This process would make $z(t)$ arbitrarily small. The controller of [7] has a switching nature and the control $u$ is discontinuous. In this paper, we would like to construct a feedback law whose control is continuous in time. To this end, we introduce a continuously decreasing variable $\alpha_{1}(t)$ and we attempt to make $z(t)$ approach $\alpha_{1}(t) V w$.
Our controller takes the following form:

$$
\begin{aligned}
u & =g(\alpha, z, w)=\left(1-\alpha_{1}\right) \Gamma w+\alpha_{2} f\left(\frac{z-\alpha_{1} V w}{\alpha_{2}}\right) \\
\dot{\alpha}_{1} & = \begin{cases}0, & \text { if } \frac{z-\alpha_{1} V w}{\alpha_{2}} \in \mathcal{S} \backslash \mathcal{S}_{I} \\
-\gamma \alpha_{1}, & \text { if } \frac{z-\alpha_{1} V w}{\alpha_{2}} \in \mathcal{S}_{I}\end{cases} \\
\dot{\alpha}_{2} & = \begin{cases}0, & \text { if } \frac{z-\alpha_{1} V w}{\alpha_{2}} \in \mathcal{S} \backslash \mathcal{S}_{I} \text { or } \alpha_{2} \leq \delta \\
-\gamma \alpha_{2}, & \text { if } \frac{z-\alpha_{1} V w}{\alpha_{2}} \in \mathcal{S}_{I} \text { and } \alpha_{2}>\delta\end{cases} \\
\alpha_{1}(0) & =1 \quad \alpha_{2}(0)=1
\end{aligned}
$$

where the value of the parameter $\gamma>0$ is to be specified shortly. We see that the state $\alpha_{2}$ is introduced to avoid singularity. From the state equations, it can be seen that $\alpha_{2}(t) \geq \delta$ for all $t>0$. The control $u$ is continuous in time since $\alpha_{1}(\cdot), \alpha_{2}(\cdot)$ and $f(\cdot)$ are continuous.

Define

$$
\gamma_{0}:=\inf _{w \in \mathcal{W}_{0}, v \in \mathcal{S}_{I}} \frac{d_{0}}{|V w|_{\infty}+|v|_{\infty}} .
$$

Since $\mathcal{W}_{0}$ and $\mathcal{S}_{I}$ are bounded, $\gamma_{0}>0$.

Theorem 2: Consider the connection of the system (15) with the controller (22). Let $\gamma$ be chosen from $\left(0, \gamma_{0}\right]$. Then, for all $\left(z_{0}, w_{0}\right) \in D_{z w}$,

$$
\lim _{t \rightarrow \infty} z\left(t, z_{0}, w_{0}\right)=0
$$

i.e., $D_{z w} \subset \mathcal{S}_{z w}$.

Proof: For the closed-loop system

$$
\dot{z}=A z+\alpha_{2} B f\left(\frac{z-\alpha_{1} V w}{\alpha_{2}}\right)-\alpha_{1} B \Gamma w .
$$

Applying (19), we obtain

$$
\begin{aligned}
\dot{z} & =A z+\alpha_{2} B f\left(\frac{z-\alpha_{1} V w}{\alpha_{2}}\right)-\alpha_{1} A V w+\alpha_{1} V S w \\
& =A\left(z-\alpha_{1} V w\right)+\alpha_{2} B f\left(\frac{z-\alpha_{1} V w}{\alpha_{2}}\right)+\alpha_{1} V \dot{w}
\end{aligned}
$$

which can be rewritten as

$$
\frac{\dot{z}-\alpha_{1} V \dot{w}}{\alpha_{2}}=A\left(\frac{z-\alpha_{1} V w}{\alpha_{2}}\right)+B f\left(\frac{z-\alpha_{1} V w}{\alpha_{2}}\right) .
$$

Let $v=\left(z-\alpha_{1} V w\right) / \alpha_{2}$, we have

$$
\begin{aligned}
\dot{v} & =\frac{\dot{z}-\dot{\alpha}_{1} V w-\alpha_{1} V \dot{w}}{\alpha_{2}}-\frac{z-\alpha_{1} V w}{\alpha_{2}^{2}} \dot{\alpha}_{2} \\
& =\frac{\dot{z}-\alpha_{1} V \dot{w}}{\alpha_{2}}-\left(\frac{\dot{\alpha}_{1}}{\alpha_{2}}\right) V w-\left(\frac{\dot{\alpha}_{2}}{\alpha_{2}}\right) v \\
& =A v+B f(v)-\left(\frac{\dot{\alpha}_{1}}{\alpha_{2}}\right) V w-\left(\frac{\dot{\alpha}_{2}}{\alpha_{2}}\right) v .
\end{aligned}
$$

Now, suppose that $\left(z_{0}, w_{0}\right) \in D_{z w}$. Since $\alpha_{1}(0)=\alpha_{2}(0)=$ 1 , we have $v(0)=z_{0}-V w_{0} \in \mathcal{S}$. If $v(0) \in \mathcal{S} \backslash \mathcal{S}_{I}$, then $\dot{\alpha}_{1}=\dot{\alpha}_{2}=0$, and we have

$$
\dot{v}=A v+B f(v) .
$$

By assumption, the origin of this system has a domain of attraction $\mathcal{S}$. Since $v(0) \in \mathcal{S}$ and $\mathcal{S}_{I}$ is a neighborhood of the origin, 
$v(t)$ will enter $\mathcal{S}_{I}$ at some $t=t_{0}$. By this time, we still have $\alpha_{1}\left(t_{0}\right)=\alpha_{2}\left(t_{0}\right)=1$. For $t \geq t_{0}$

$$
\dot{\alpha}_{1}=-\gamma \alpha_{1} \quad \dot{\alpha}_{2}=-\gamma \alpha_{2}
$$

and $\alpha_{1}=\alpha_{2}$ as long as $\alpha_{2}>\delta$. Now, the dynamic of the system is

$$
\dot{v}=A v+B f(v)+\gamma(V w+v) .
$$

Since $\gamma \in\left(0, \gamma_{0}\right)$, we have

$$
|\gamma(V w+v)|_{\infty} \leq d_{0}
$$

for all $w \in \mathcal{W}_{0}$ and $v \in \mathcal{S}_{I}$. By assumption, $\mathcal{S}_{I}$ is an invariant set for the system (24) and hence we have $v(t) \in \mathcal{S}_{I}$ for all $t \geq t_{0}$.

As $t$ increases, $\alpha_{1}=\alpha_{2}$ will decrease until $\alpha_{2}=\delta$. Let the time when $\alpha_{2}$ first reaches $\delta$ be $t_{1}$, then for $t>t_{1}$, we have $\dot{\alpha}_{2}=0, \alpha_{2}=\delta, \alpha_{1}<\delta$, and

$$
\dot{v}=A v+B f(v)+\gamma\left(\frac{\alpha_{1}}{\delta}\right) V w .
$$

Since $\left|\gamma\left(\alpha_{1} / \delta\right) V w\right|_{\infty} \leq d_{0}$ and $\lim _{t \rightarrow \infty} \alpha_{1}=0$, by assumption on the stabilizing controller, we have

$$
\lim _{t \rightarrow \infty} v(t)=0 .
$$

Note that for $t>t_{1}$

$$
v=\frac{z-\alpha_{1} V w}{\delta} .
$$

It follows that $\lim _{t \rightarrow \infty} z(t)=0$.

Finally, it is straightforward to verify that

$$
g(\alpha, z, w) \in \mathcal{U}
$$

for all $t \geq 0\left(t \in\left[0, t_{0}\right],\left[t_{0}, t_{1}\right],\left[t_{1}, \infty\right]\right.$ respectively, noting that $\Gamma w \in(1-\delta) \mathcal{U}$ and $f(v) \in \mathcal{U})$. Therefore, $\lim _{t \rightarrow \infty} z(t)=0$ for all $\left(z_{0}, w_{0}\right) \in D_{z w}$, i.e., $D_{z w} \subset \mathcal{S}_{z w}$.

\section{ERROR FEEDBACK}

Consider again the open-loop system (4). Here in this section, we assume that only the error $e=C z$ is available for feedback. Also, without loss of generality, assume that the pair

$$
(\bar{C}, \bar{A})=\left(\left[\begin{array}{ll}
C & 0
\end{array}\right],\left[\begin{array}{cc}
A & -B \Gamma \\
0 & S
\end{array}\right]\right)
$$

is observable. If it is detectable, but not observable, then the unobservable modes must be the asymptotically stable eigenvalues of $A$, which do not affect the output regulation [see (4)] and, hence, can be left out.

Our controller consists of an observer and a state feedback law which is based on a stabilizing feedback law $u=f(v)$. Because of the observer error, we need an additional assumption on $f(v)$ so that some class of disturbances can be tolerated. Consider the system

$$
\dot{v}=A v+B f\left(v+\eta_{1}\right)+\eta_{2}
$$

where $f: \mathbf{R}^{n} \rightarrow \mathcal{U}$, and $\eta_{1}$ and $\eta_{2}$ stand for the disturbance arising from, for example, the observer error. Assume that $f(\cdot)$ is continuous and the following conditions are satisfied.
C1) For the case where $\eta_{2} \equiv 0$, there exist a set $D_{0} \subset \mathbf{R}^{n}$ and positive numbers $k$ and $d_{1}$ such that the solution of (25) satisfies

$$
\begin{aligned}
\|v\|_{\infty} & \leq k \max \left(\left|v_{0}\right|_{\infty},\left\|\eta_{1}\right\|_{\infty}\right), \\
\|v\|_{a} & \leq k\left\|\eta_{1}\right\|_{a} \quad \forall v_{0} \in D_{0},\left\|\eta_{1}\right\|_{\infty} \leq d_{1}
\end{aligned}
$$

where $\|v\|_{a}=\lim \sup _{t \rightarrow \infty}|v(t)|_{\infty}$.

C2) For the case where $\eta_{1} \equiv 0$, there exists a $d_{2}>0$ and a set $\mathcal{S}_{I}$ containing the origin in its interior, such that $\mathcal{S}_{I}$ is invariant for all $\left\|\eta_{2}\right\|_{\infty} \leq d_{2}$. Moreover, for every $v_{0} \in \mathcal{S}_{I}, v(t) \rightarrow 0$ as long as $\left\|\eta_{2}\right\|_{\infty} \leq d_{2}$ and $\eta_{2}(t) \rightarrow 0$.

When the condition $\mathrm{C} 1$ ) is true, the system is said to satisfy an asymptotic bound from $D_{0}$ with gain $k$ and restriction $d_{1}$ [27]. In this paper, the condition is imposed to ensure that $v$ is bounded and as $\eta_{1} \rightarrow 0$, we have $v \rightarrow 0$. Condition C2) is imposed for the continuity of the feedback law, as in Section IV. For $D_{0}$, we would like to make it as large as possible. For $\mathcal{S}_{I}$, since its size will affect the overall convergence rate, it should not be too large.

Remark 3: In [8], a saturated linear feedback $u=$ $f(v)=\operatorname{sat}(F v)$ satisfying condition $\mathrm{C} 1)$ is constructed for second-order antistable systems and the set $D_{0}$ can be made arbitrarily close to the null controllable region $\mathcal{C}$. Since the feedback law is linear in a local region, there always exist $d_{2}>0$ and $\mathcal{S}_{I}$ satisfying condition C2).

For more general systems, we can use the method in [9] to design a saturated linear feedback law along with a set $D_{0}$ (as large as possible) to satisfy condition C1). Although this method was developed for disturbances of the form $\eta_{2}$, it can be easily adapted for dealing with disturbances of the form $\eta_{1}$, which can be transformed into the form of $\eta_{2}$ in a bounded region of the state space. Also, since the feedback law is linear in a local region, there always exist $d_{2}>0$ and $\mathcal{S}_{I}$ satisfying condition C2).

For the feedback laws constructed in [9] and [8], the gain $k$ in condition $\mathrm{C} 1$ ) can be estimated. However, we will not discuss how to estimate this gain since it will not be used in this paper.

We use the following observer to reconstruct the states $z$ and $w$,

$$
\begin{aligned}
\dot{\bar{z}} & =A \bar{z}+B u-B \Gamma \bar{w}-L_{1}(e-C \bar{z}) \\
\dot{\bar{w}} & =S \bar{w}-L_{2}(e-C \bar{z}) .
\end{aligned}
$$

Letting $\tilde{z}=z-\bar{z}, \tilde{w}=w-\bar{w}$, we can write the composite system as

$$
\begin{aligned}
\dot{z} & =A z+B u-B \Gamma w \\
\dot{w} & =S w \\
{\left[\begin{array}{c}
\dot{\tilde{z}} \\
\dot{\tilde{w}}
\end{array}\right] } & =\left[\begin{array}{cc}
A+L_{1} C & -B \Gamma \\
L_{2} C & S
\end{array}\right]\left[\begin{array}{c}
\tilde{z} \\
\tilde{w}
\end{array}\right] .
\end{aligned}
$$

Now, we have to use $(\bar{z}, \bar{w})$ instead of $(z, w)$ to construct a feedback controller. Since $(\bar{C}, \bar{A})$ is observable, we can choose $L=\left[\begin{array}{l}L_{1} \\ L_{2}\end{array}\right]$ appropriately such that the estimation error $(\tilde{z}, \tilde{w})$ decays arbitrarily fast. Moreover, the following fact is easy to establish. 
Lemma 2: Denote

$$
\tilde{A}=\left[\begin{array}{cc}
A+L_{1} C & -B \Gamma \\
L_{2} C & S
\end{array}\right] .
$$

Given any (arbitrarily small) positive numbers $T$ and $\varepsilon$, there exists an $L$ such that

$$
\max \left\{\left|e^{\tilde{A} t}\right|,|L| \cdot\left|e^{\tilde{A} t}\right|\right\} \leq \varepsilon \quad \forall t \geq T
$$

where $|\cdot|$ can be any matrix norm.

Because of this lemma, it is expected that the controller based on the observer can achieve almost the same performance as the state feedback controller.

Let $D \in \mathbf{R}^{n}$ be in the interior of $D_{0}$, i.e., the distance from any point in $D$ to the boundary of $D_{0}$ is greater than a fixed positive number. Given a number $M$, denote

$$
\begin{aligned}
& D_{M}=\left\{(z, w, \tilde{z}, \tilde{w}) \in \mathbf{R}^{n} \times \mathcal{W}_{0} \times \mathbf{R}^{n} \times \mathbf{R}^{r}:\right. \\
&\left.z-V w \in D,\left|\left[\begin{array}{c}
\tilde{z} \\
\tilde{w}
\end{array}\right]\right| \leq M\right\} .
\end{aligned}
$$

It is clear that the set $D_{M}$ increases as $D$ increases. From Theorem 1 , we see that if $D=\mathcal{C}^{a}$, then the projection of $D_{M}$ to the $(z, w)$-subspace equals to $\mathcal{R}_{g}^{a}$. To enlarge the set of initial conditions where output regulation is achieved, it suffices to construct a state feedback law $u=f(v)$ to enlarge $D_{0}$, choose a set $D$ very close to $D_{0}$ and design an observer such that for all $\left(z_{0}, w_{0}, \tilde{z}_{0}, \tilde{w}_{0}\right) \in D_{M}, \lim _{t \rightarrow \infty} z(t)=0$. The objective in this section is to construct such an observer along with a feedback law, given $f(\cdot), D_{0}$ and $D$ in the interior of $D_{0}$.

Consider (27). For simplicity, we also assume that there exists a matrix $V$ that satisfies

$$
-A V+V S=-B \Gamma
$$

Letting $v=z-V w$, we obtain $\dot{v}=A v+B u$. Suppose that $\left(z_{0}, w_{0}, \tilde{z}_{0}, \tilde{w}_{0}\right) \in D_{M}$, then $v_{0}=z_{0}-V w_{0} \in D$. Since $D$ is in the interior of $D_{0}$, there exists a $T_{0}>0$ such that, with any admissible control $u(t) \in \mathcal{U}$, we have

$$
v(t)=z(t)-V w(t) \in D_{0} \quad \forall \in\left[0, T_{0}\right] .
$$

What we are going to do is to choose an $L$ such that the estimation error is sufficiently small after $T_{0}$, and to design a feedback law to make $z(t)-\alpha_{1}(t) V w(t) \rightarrow 0$ with $\alpha_{1}(t) \rightarrow 0$.

Lemma 3: There exists an $L \in \mathbf{R}^{(n+r) \times q}$ such that, under the control

$$
u=f(\bar{z}-V \bar{w})
$$

the solution of (27) satisfies

$$
\lim _{t \rightarrow \infty}(z(t)-V w(t))=0 \quad \forall\left(z_{0}, w_{0}, \tilde{z}_{0}, \tilde{w}_{0}\right) \in D_{M}
$$

Proof: Let $v=z-V w$. Since $u(t)=f(\bar{z}(t)-V \bar{w}(t)) \in$ $\mathcal{U}$, there exists a $T_{0}$ such that

$$
v(t)=z(t)-V w(t) \in D_{0} \quad \forall t \in\left[0, T_{0}\right] .
$$

Let $\tilde{v}=\tilde{z}-V \tilde{w}$, by Lemma 2 , there exists an $L \in \mathbf{R}^{(n+r) \times q}$ such that for all $\left(z_{0}, w_{0}, \tilde{z}_{0}, \tilde{w}_{0}\right) \in D_{M}$

$$
|\tilde{v}(t)|_{\infty}=|\tilde{z}(t)-V \tilde{w}(t)|_{\infty} \leq d_{1} \quad \forall t \geq T_{0} .
$$

We now consider the system after $T_{0}$. For $t \geq T_{0}$, the closedloop system is

$\dot{v}=A v+B f(\bar{z}-V \bar{w})=A v+B f(v-\tilde{v}), \quad v\left(T_{0}\right) \in D_{0}$.

By condition $\mathrm{C} 1$ ), this system satisfies an asymptotic bound from $D_{0}$ with a finite gain and restriction $d_{1}$. It follows from (30), (31), and $\lim _{t \rightarrow \infty} \tilde{v}(t)=0$ that

$$
\lim _{t \rightarrow \infty}(z(t)-V w(t))=\lim _{t \rightarrow \infty} v(t)=0 .
$$

Lemma 3 means that we can keep $z(t)$ bounded if $\left(z_{0}, w_{0}, \tilde{z}_{0}, \tilde{w}_{0}\right) \in D_{M}$. Just as the state feedback case, we want to move $z(t)$ to the origin by making $z(t)-\alpha_{1}(t) V w(t) \rightarrow 0$ with $\alpha_{1}(t) \rightarrow 0$. Since the feedback has to be based on $(\bar{z}, \bar{w})$, we will cause $z(t)-\alpha_{1}(t) V w(t) \rightarrow 0$ by driving $\bar{z}(t)-\alpha_{1}(t) V \bar{w}(t) \rightarrow 0$.

Our control law based on the state of the observer is as follows:

$$
\begin{gathered}
u=g(\alpha, \bar{z}, \bar{w})=\left(1-\alpha_{1}\right) \Gamma \bar{w}+\alpha_{2} f\left(\frac{\bar{z}-\alpha_{1} V \bar{w}}{\alpha_{2}}\right), \\
\dot{\alpha}_{1}= \begin{cases}0, & \text { if } \frac{\bar{z}-\alpha_{1} V \bar{w}}{\alpha_{2}} \in \mathcal{S} \backslash \mathcal{S}_{I} \\
-\gamma \alpha_{1}, & \text { if } \frac{\bar{z}-\alpha_{1} V \bar{w}}{\alpha_{2}} \in \mathcal{S}_{I}\end{cases} \\
\dot{\alpha}_{2}= \begin{cases}0, & \text { if } \frac{\bar{z}-\alpha_{1} V \bar{w}}{\alpha_{2}} \in \mathcal{S} \backslash \mathcal{S}_{I} \text { or } \alpha_{2} \leq \delta \\
-\gamma \alpha_{2}, & \text { if } \frac{\bar{z}-\alpha_{1} V \bar{w}}{\alpha_{2}} \in \mathcal{S}_{I} \text { and } \alpha_{2}>\delta\end{cases} \\
\alpha_{1}(t)=1 \quad \alpha_{2}(t)=1, t \in\left[0, T_{0}\right]
\end{gathered}
$$

where the value of the parameter $\gamma$ is to be specified.

Define

$$
\gamma_{0}:=\inf _{w \in \mathcal{W}_{0}, v \in \mathcal{S}_{I}} \frac{d_{2}}{2\left(|V w|_{\infty}+|v|_{\infty}\right)} .
$$

Theorem 3: Let $\gamma$ be chosen from $\left(0, \gamma_{0}\right]$. By Lemma 2 , there exists an $L$ such that for all $\left(z_{0}, w_{0}, \tilde{z}_{0}, \tilde{w}_{0}\right) \in D_{M}$ and $t \geq T_{0}$

$$
|\tilde{z}(t)-V \tilde{w}(t)|_{\infty} \leq d_{1}
$$

and

$$
|\gamma V \tilde{w}|_{\infty}+\frac{\left(\left|L_{1} C \tilde{z}(t)\right|_{\infty}+\left|V L_{2} C \tilde{z}(t)\right|_{\infty}\right)}{\delta} \leq \frac{d_{2}}{2}
$$

Consider the connection of the system (27) and the controller (32) with $L$ thus chosen. Then, for all $\left(z_{0}, w_{0}, \tilde{z}_{0}, \tilde{w}_{0}\right) \in D_{M}$

$$
\lim _{t \rightarrow \infty} z(t)=0 \text {. }
$$

Proof: Under the control law (32), we have

$$
\begin{aligned}
\dot{\bar{z}}= & A \bar{z}+B\left(1-\alpha_{1}\right) \Gamma \bar{w}+B \alpha_{2} f\left(\frac{\bar{z}-\alpha_{1} V \bar{w}}{\alpha_{2}}\right) \\
& -B \Gamma \bar{w}-L_{1} C \tilde{z} \\
= & A \bar{z}-\alpha_{1} B \Gamma \bar{w}+\alpha_{2} B f\left(\frac{\bar{z}-\alpha_{1} V \bar{w}}{\alpha_{2}}\right)-L_{1} C \tilde{z} .
\end{aligned}
$$


Replacing $-B \Gamma$ with $-A V+V S$, we have

$$
\dot{\bar{z}}=A \bar{z}-\alpha_{1} A V \bar{w}+\alpha_{1} V S \bar{w}+\alpha_{2} B f\left(\frac{\bar{z}-\alpha_{1} V \bar{w}}{\alpha_{2}}\right)-L_{1} C \tilde{z} .
$$

Recalling that $S \bar{w}=\dot{\bar{w}}+L_{2} C \tilde{z}$, we obtain

$$
\begin{aligned}
\dot{\bar{z}}-\alpha_{1} V \dot{\bar{w}}=A\left(\bar{z}-\alpha_{1} V \bar{w}\right)+\alpha_{2} B f\left(\frac{\bar{z}-\alpha_{1} V \bar{w}}{\alpha_{2}}\right) \\
-\left(L_{1}-\alpha_{1} V L_{2}\right) C \tilde{z} .
\end{aligned}
$$

If we define $v=\left(\bar{z}-\alpha_{1} V \bar{w}\right) / \alpha_{2}$, then similar to the proof of Theorem 2

$$
\begin{aligned}
\dot{v}=A v+B f(v)- & \left(\frac{\dot{\alpha}_{1}}{\alpha_{2}}\right) V w-\left(\frac{\dot{\alpha}_{2}}{\alpha_{2}}\right) v \\
& +\left(\frac{\dot{\alpha}_{1}}{\alpha_{2}}\right) V \tilde{w}-\frac{L_{1}-\alpha_{1} V L_{2}}{\alpha_{2}} C \tilde{z} .
\end{aligned}
$$

For $t \in\left[0, T_{0}\right], \alpha_{1}(t)=\alpha_{2}(t)=1$ and $u=f(\bar{z}-V \bar{w})$. If $v\left(T_{0}\right) \notin \mathcal{S}_{I}$, then $\alpha_{1}$ and $\alpha_{2}$ will stay at the value 1 and the control will continue to be $u=f(\bar{z}-V \bar{w})$. By Lemma 3 , we will have $\lim _{t \rightarrow \infty}(z(t)-V w(t))=0$. Since $\lim _{t \rightarrow \infty} \tilde{z}=0$ and $\lim _{t \rightarrow \infty} \tilde{w}=0$ and $\mathcal{S}_{I}$ contains the origin in its interior, there will be a finite time $t_{1} \geq T_{0}$ such that $v\left(t_{1}\right)=\bar{z}\left(t_{1}\right)-V \bar{w}\left(t_{1}\right) \in$ $\mathcal{S}_{I}$. After $t_{1}, \alpha_{1}$ and $\alpha_{2}$ will start to decrease and $\alpha_{1} \leq \alpha_{2}$ for all $t \geq t_{1}$. Since $\gamma \in\left(0, \gamma_{0}\right)$, it can be verified that

$$
\left|\frac{\dot{\alpha}_{1}(t)}{\alpha_{2}(t)}\right| \leq\left|\frac{\dot{\alpha}_{1}(t)}{\alpha_{1}(t)}\right| \leq \gamma_{0} \quad\left|\frac{\dot{\alpha}_{2}(t)}{\alpha_{2}(t)}\right| \leq \gamma_{0}
$$

for all $t \geq t_{1}$. Hence

$$
\left|\frac{\dot{\alpha}_{1}(t)}{\alpha_{2}(t)} V w(t)+\frac{\dot{\alpha}_{2}(t)}{\alpha_{2}(t)} v(t)\right|_{\infty} \leq \frac{d_{2}}{2} \quad \forall t>t_{1} .
$$

By the choice of $L$, we have

$$
\left|\frac{\dot{\alpha}_{1}(t)}{\alpha_{2}(t)} V \tilde{w}(t)-\frac{L_{1}-\alpha_{1}(t) V L_{2}}{\alpha_{2}} C \tilde{z}(t)\right|_{\infty} \leq \frac{d_{2}}{2} \quad \forall t>t_{1} .
$$

By condition C2), $\mathcal{S}_{I}$ will be an invariant set for $t>t_{1}$ and as $t \rightarrow \infty, \alpha_{2} \rightarrow \delta, \dot{\alpha}_{1} / \alpha_{2}, \dot{\alpha}_{2} / \alpha_{2}, \tilde{z} \rightarrow 0$, hence, the term

$$
-\left(\frac{\dot{\alpha}_{1}}{\alpha_{2}}\right) V w-\left(\frac{\dot{\alpha}_{2}}{\alpha_{2}}\right) v+\left(\frac{\dot{\alpha}_{1}}{\alpha_{2}}\right) V \tilde{w}-\frac{L_{1}-\alpha_{1} V L_{2}}{\alpha_{2}} C \tilde{z}
$$

will tend to zero. It follows that $v$ will converge to zero and so will $\bar{z}-\alpha_{1} \bar{w}, \bar{z}$ and $z$.

The error feedback law is constructed on a stabilizing feedback law $u=f(v)$ satisfying conditions $\mathrm{C} 1$ ) and $\mathrm{C} 2$ ). So far, we have assumed that the system model is accurate. To account for the model uncertainties, we can impose further requirements on the sets $D_{0}$ and $\mathcal{S}_{I}$, specifically, that they are both strictly invariant. For $D_{0}$, being strictly invariant implies that $\dot{v}=A v+B f\left(v+\eta_{1}\right)$ points strictly inward of the boundary of $D_{0}$ for all $v \in \partial D_{0}$ and $\left\|\eta_{1}\right\|_{\infty} \leq d_{1}$. For $\mathcal{S}_{I}$, being strictly invariant implies that $\dot{v}=A v+B \bar{f}(v)+\eta_{2}$ points strictly inward of the boundary of $\mathcal{S}_{I}$ for all $v \in \partial \mathcal{S}_{I}$ and $\left\|\eta_{2}\right\|_{\infty} \leq d_{2}$. These strict invariance properties will guarantee that the sets are invariant in the presence of small parameter perturbations and will also guarantee a certain convergence rate.
The design methods in [8] and [9] can also be used to construct such strictly invariant sets $D_{0}$ and $\mathcal{S}_{I}$. Actually, in [8], the set $D_{0}$ is already an invariant set and it can be shown that for $\varepsilon>0$ sufficiently small, the set $(1-\varepsilon) D_{0}$ is strictly invariant.

This paper will not pursue the exact characterization of the amount of uncertainty that the system can tolerate but will use simulation results to demonstrate this aspect.

\section{NUMERICAL EXAMPLE AND SOME ROBUSTNESS ISSUES}

\section{A. Model}

In this section, we apply the results developed in Sections IV and $\mathrm{V}$ to the control of an aircraft model. Consider the longitudinal dynamics of the TRANS3 aircraft under certain flight condition [17]

$$
\begin{aligned}
& \dot{x}=A x+B u+P w \\
& y=C x
\end{aligned}
$$

with

$$
\begin{aligned}
A & =\left[\begin{array}{rrrr}
0 & 14.3877 & 0 & -31.5311 \\
-0.0012 & -0.4217 & 1.0000 & -0.0284 \\
0.0002 & -0.3816 & -0.4658 & 0 \\
0 & 0 & 1.0000 & 0
\end{array}\right] \\
B & =0.1745 \times\left[\begin{array}{rr}
4.526 \\
-0.0337 \\
-1.4566 \\
0
\end{array}\right] \\
P & =\left[\begin{array}{rrrr}
-0.6526 & -0.3350 & 0.4637 & 0.9185 \\
0.0049 & 0.0025 & -0.0035 & -0.0068 \\
0.2100 & 0.1078 & -0.1492 & -0.2956 \\
0 & 0 & 0 & 0
\end{array}\right]
\end{aligned}
$$

and $C=\left[\begin{array}{llll}1 & 0 & 0 & 0\end{array}\right]$, where the state consists of the velocity $x_{1}$ (feet/s, relative to the nominal flight condition), the angle of attack $x_{2}$ (degree), the pitch rate $x_{3}$ (degree/s) and the Euler angle rotation of aircraft about the inertial $y$-axis $x_{4}$ (degree), the control $u$ (degree) is the elevator input, whose value is scaled to between \pm 1 (corresponding to $\pm 10^{\circ}$ ). Hence, the control constraint set is $\mathcal{U}=[-1,1]$. The design objective is to reject the disturbance $P w$, where $w$ has two frequency component of $0.1 \mathrm{rad} / \mathrm{s}$ and $0.3 \mathrm{rad} / \mathrm{s}$. Clearly, this problem can be cast into an output regulation problem for (1) with

$$
S=\left[\begin{array}{rrrr}
0 & -0.1 & 0 & 0 \\
0.1 & 0 & 0 & 0 \\
0 & 0 & 0 & -0.3 \\
0 & 0 & 0.3 & 0
\end{array}\right]
$$

and $Q=0$. A solution to the linear matrix (2) is

$$
\Pi=0 \quad \Gamma=\left[\begin{array}{llll}
0.8263 & 0.4242 & -0.5871 & -1.1630
\end{array}\right] .
$$

Assume that the disturbances are bounded by $\|\Gamma w\|_{\infty} \leq \rho=$ 0.9. Thus, $\delta=0.1$.

The matrix $A$ has two stable eigenvalues $-0.4650 \pm 0.6247 i$ and two antistable ones, $0.0212 \pm 0.1670 i$. With state transformation, we obtain the matrices for the antistable subsystem

$$
A_{2}=\left[\begin{array}{cc}
0.0212 & 0.1670 \\
-0.1670 & 0.0212
\end{array}\right] \quad B_{2}=\left[\begin{array}{c}
8.2856 \\
-2.4303
\end{array}\right] \text {. }
$$


For the state feedback case, we don't need to worry about the exponentially stable $z_{1}$-subsystem since its state is bounded under any bounded input $u-\Gamma w$ and will converge to the origin as the combined input goes to zero. Therefore, we only need to consider the problem of output regulation for the antistable subsystem

$$
\begin{aligned}
\dot{z}_{2} & =A_{2} z_{2}+B_{2} u-B_{2} \Gamma w \\
\dot{w} & =S w .
\end{aligned}
$$

A state feedback controller for this subsystem will also work for the original system.

The solution $V_{2}$ to $-A_{2} V_{2}+V_{2} S=-B_{2} \Gamma$ is

$$
V_{2}=\left[\begin{array}{llll}
17.8341 & 46.6790 & -46.5057 & 25.0374 \\
66.6926 & 4.4420 & 21.7554 & 22.6661
\end{array}\right]
$$

\section{B. State Feedback Law}

We first construct a stabilizing feedback law $u=f(v)$ for the antistable system

$$
\dot{v}=A_{2} v+B_{2} u \text {. }
$$

For $\varepsilon>0$, let $X$ be the solution to the algebraic Riccati equation

$$
A_{2}^{T} X+X A_{2}-X B_{2} B_{2}^{T} X+\varepsilon I=0
$$

and let $F_{0}(\varepsilon)=-B_{2}^{T} X$. It was shown in [8] that as $\varepsilon \rightarrow 0$ and $k \rightarrow \infty$, the domain of attraction of the origin of the system

$$
\dot{v}=A_{2} v+B_{2} \operatorname{sat}\left(F_{2} v\right) \quad F_{2}=k F_{0}(\varepsilon)
$$

will approach the null controllable region of the system (36). To ensure some capability of disturbance rejection, $\varepsilon$ should be greater than zero. Here, by choosing $\varepsilon=0.02$ and $k=1$, we obtain

$$
F_{2}=k F_{0}(\varepsilon)=\left[\begin{array}{ll}
-0.1151 & 0.1848
\end{array}\right] .
$$

The boundary of the domain of attraction, $\partial \mathcal{S}$, for the system

$$
\dot{v}=A_{2} v+B_{2} \operatorname{sat}\left(F_{2} v\right)
$$

is plotted in Fig. 1 as the larger solid curve. The outer dashed closed curve is $\partial \mathcal{C}$, the boundary of the null controllable region.

Now, we choose $u=f(v)=\operatorname{sat}\left(F_{2} v\right)$. Using the analysis method in [9], we detected an invariant set $\mathcal{S}_{I}$ for the system

$$
\begin{aligned}
\dot{v} & =A_{2} v+B_{2} f(v)+\eta \quad|\eta|_{\infty} \leq 0.5 \\
\text { where } \mathcal{S}_{I} & =\left\{v \in \mathbf{R}^{2}: v^{T} X v \leq 1\right\} \\
X & =\left[\begin{array}{cc}
0.0267 & -0.0404 \\
-0.0404 & 0.0813
\end{array}\right] .
\end{aligned}
$$

We see that $u=f(v)$ satisfies the assumptions in Section IV. In Fig. 1, the innermost closed-curve is the boundary of $\mathcal{S}_{I}$. To achieve a fast convergence rate, we have chosen a small $\mathcal{S}_{I}$ so that $\gamma_{0}$ as defined in (23) would be relatively large. The resulting $\gamma_{0}$ is 0.0085 . In the controller (22), we choose $\gamma=\gamma_{0}$.

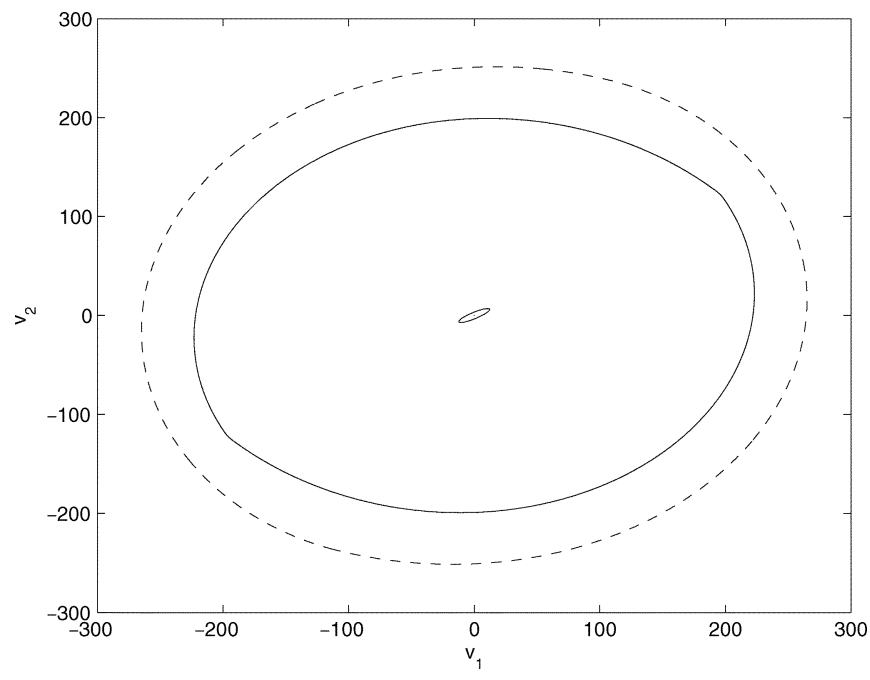

Fig. 1. Domain of attraction and an invariant set.

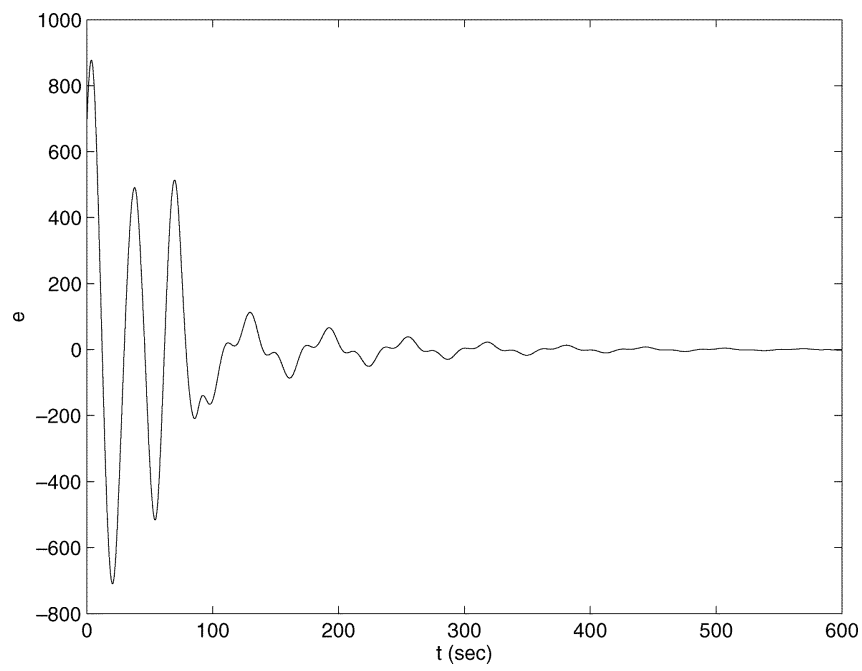

Fig. 2. Tracking error: state feedback.

In simulation, we choose an initial value $v(0)=z_{20}-V_{2} w_{0}$ which is very close to the boundary of $\mathcal{S}$, or $\left(z_{20}, w_{0}\right)$ very close to the boundary of $D_{z w}$. The tracking error is shown in Fig. 2 and the control $u$ is shown in Fig. 3. A trajectory of $v=\left(z_{2}-\alpha_{1} V_{2} w\right) / \alpha_{2}$ is plotted in Fig. 4 along with $\partial \mathcal{C}$ and $\partial \mathcal{S}$, where the initial state $v(0)$ is marked with "o."

\section{Error Feedback Law}

We next design an error feedback law. For simplicity, the initial state of the observer is set to 0 . Using the design method in Section $\mathrm{V}$, we choose $T_{0}=2 s$ and the observer gain is designed such that the eigenvalues of $\tilde{A}$ are $-17.5 \pm j 14,-17.5 \pm j 10.5$, $-17.5 \pm j 7,-17.5 \pm j 3.5$. The control law is obtained from the state feedback law by replacing $z$ and $w$ with $\bar{z}$ and $\bar{w}$, respectively. The initial conditions of the plant and the exosystem are the same as those of the state feedback case. The tracking error is shown in Fig. 5 and the control $u$ is shown in Fig. 6.

A trajectory of $v=\left(z_{2}-\alpha_{1} V_{2} w\right) / \alpha_{2}$ is plotted in Fig. 7 along with $\partial \mathcal{C}$ and $\partial \mathcal{S}$, where the initial state $v(0)$ is marked with "o." The point marked with " $*$ " is the state of $v$ at $t=$ 


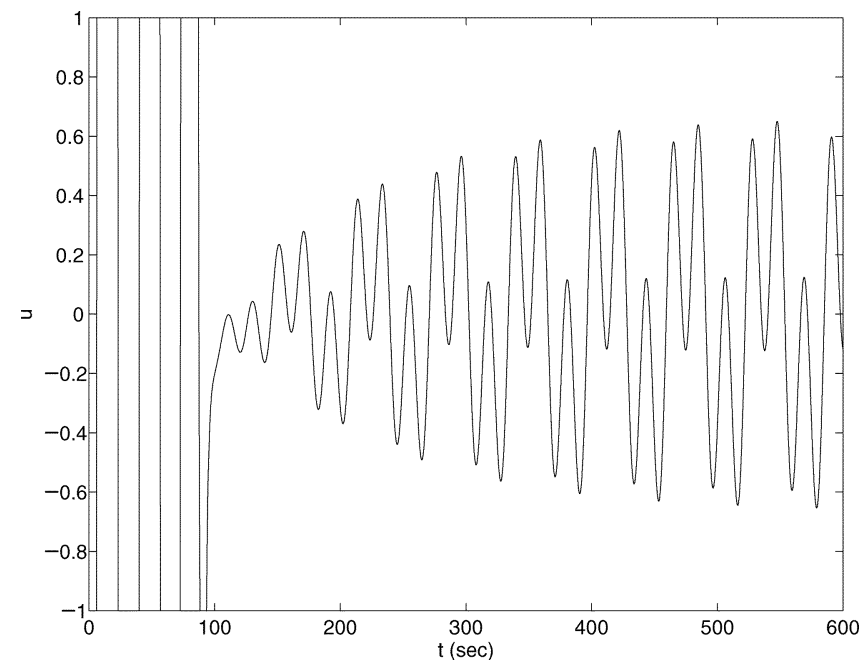

Fig. 3. Control: state feedback.

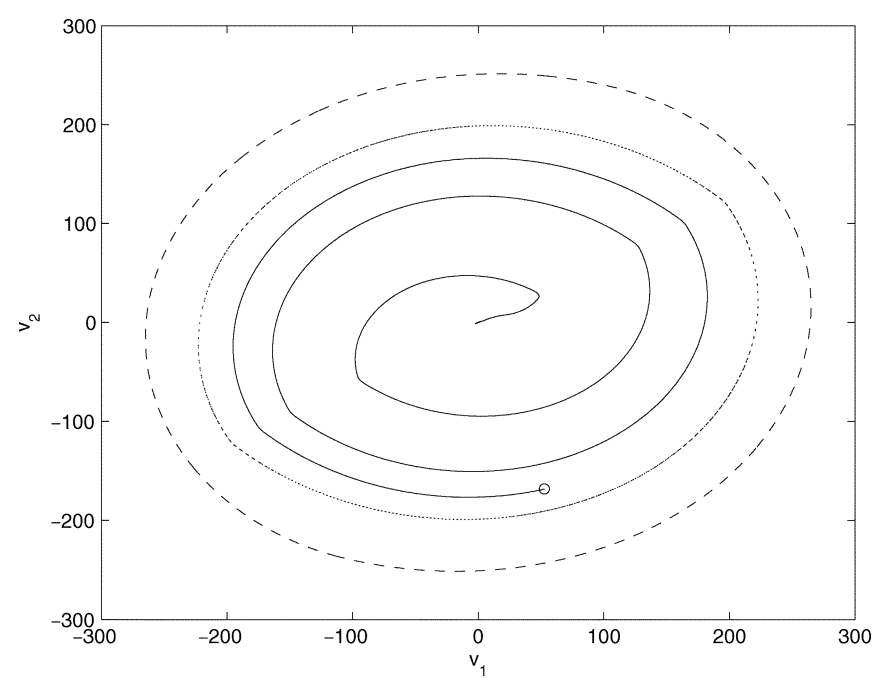

Fig. 4. Domain of attraction and a trajectory of $v$ under state feedback.

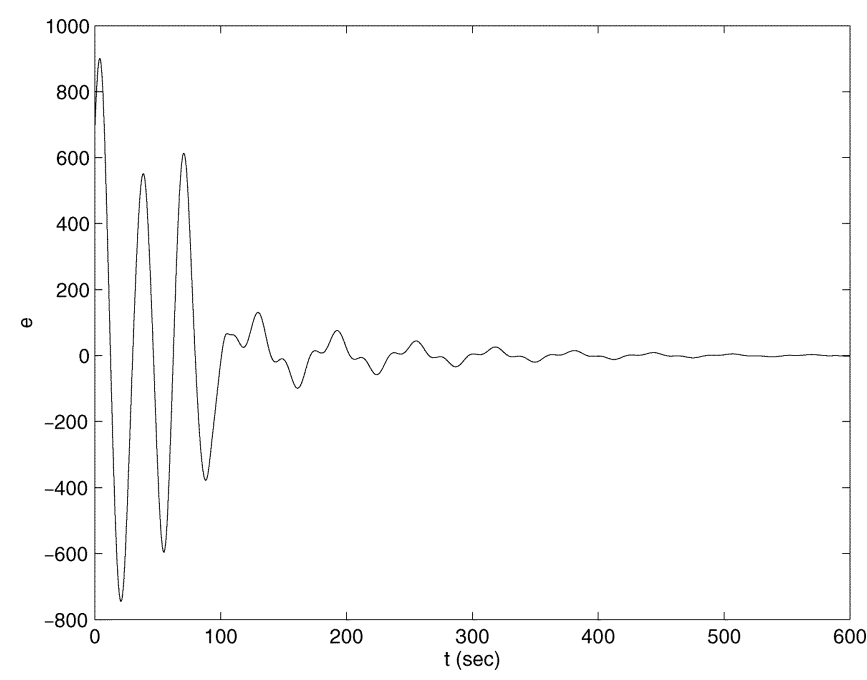

Fig. 5. Tracking error: error feedback.

$T_{0}=2 s$. For comparison, we also plotted part of the trajectory under the state feedback control in dotted curve. The estimation errors $z-\bar{z}$ and $w-\bar{w}$ are plotted in Fig. 8 for the first $2.5 \mathrm{~s}$.

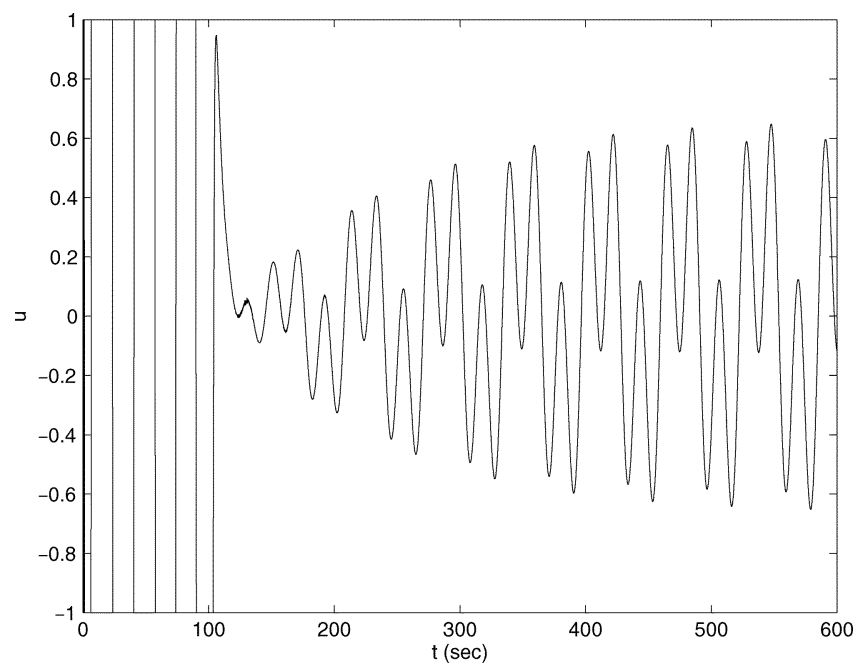

Fig. 6. Control: error feedback.

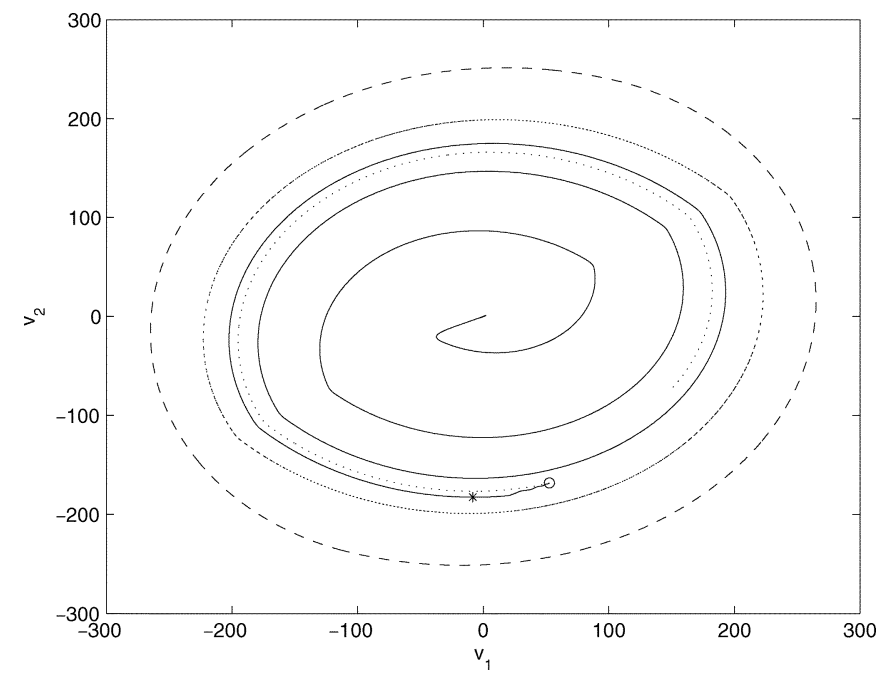

Fig. 7. Trajectory of $v$ under error feedback.

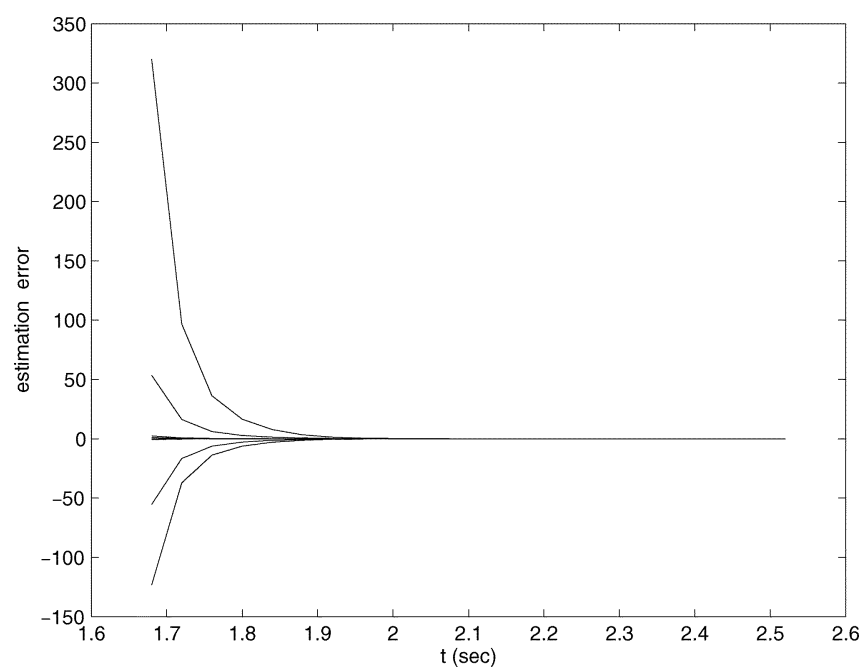

Fig. 8. Estimation error.

\section{Robustness Issues}

We demonstrate the robustness of the system under error feedback by simulation. Let $L$ and the feedback law be designed as in Section VI-C, where all the eigenvalues of $\tilde{A}$ have real 

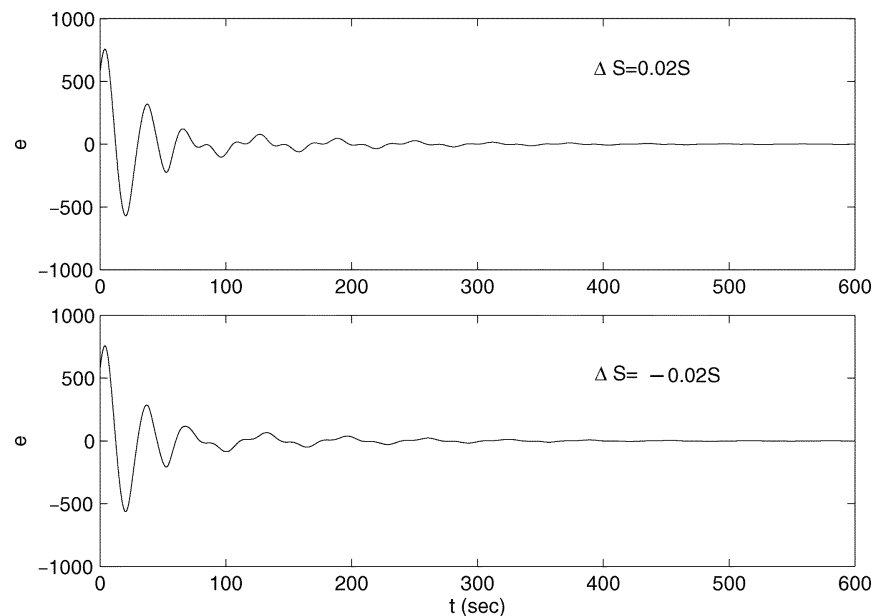

Fig. 9. Tracking errors under perturbed $S$.

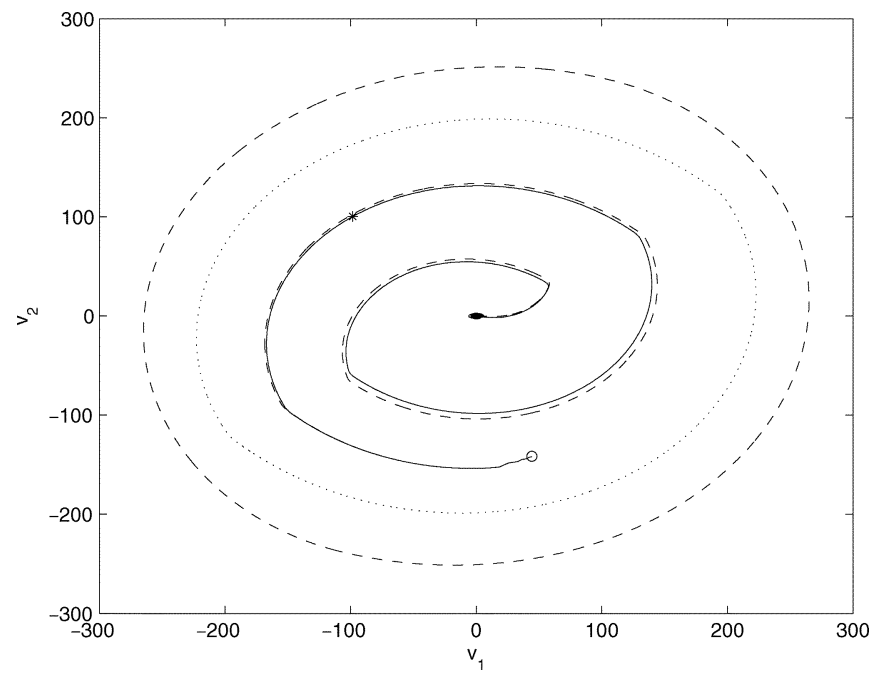

Fig. 10. Trajectories of $v$ under perturbed $S$.

part -17.5 . We first simulated the system by replacing $S$ of the exosystem with $1.02 S$ and $0.98 S$, respectively. In each of the cases, the tracking error converged to a very small interval around 0 , as plotted in Fig. 9. The two trajectories of $v$ are plotted in Fig. 10.

We next examine the robustness against measurement errors. As was shown in [5], for some type of nonlinear systems, arbitrarily small measurement noise may cause instability. For a system designed with the method in this paper, we recall that the strict invariance requirement on the sets $D_{0}$ and $\mathcal{S}_{I}$ will guarantee certain degree of robustness against parameter uncertainties and external disturbances. It is of interest to know how much robustness the system possesses. It turns out that the robustness against measurement errors is extremely weak for the current design. The output regulation can be guaranteed only for $y=(1+k) C x$, with $|k| \leq 10^{-10}$. The reason appears to be the extremely high observer gain, whose maximal element is $-1.0573 \times 10^{12}$. It seems that the high observer gain also magnifies the measurement error.
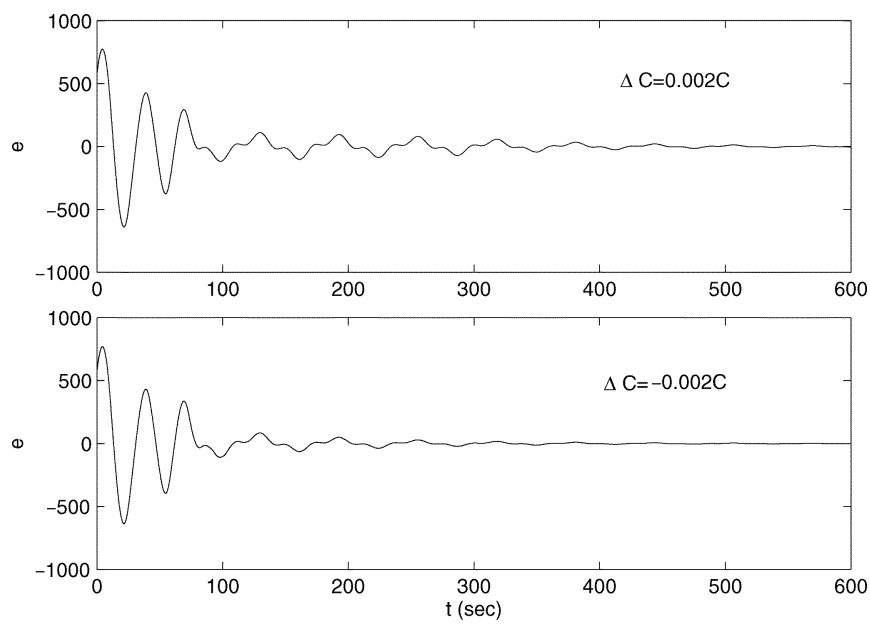

Fig. 11. Tracking errors under perturbed $C$, Case 1.
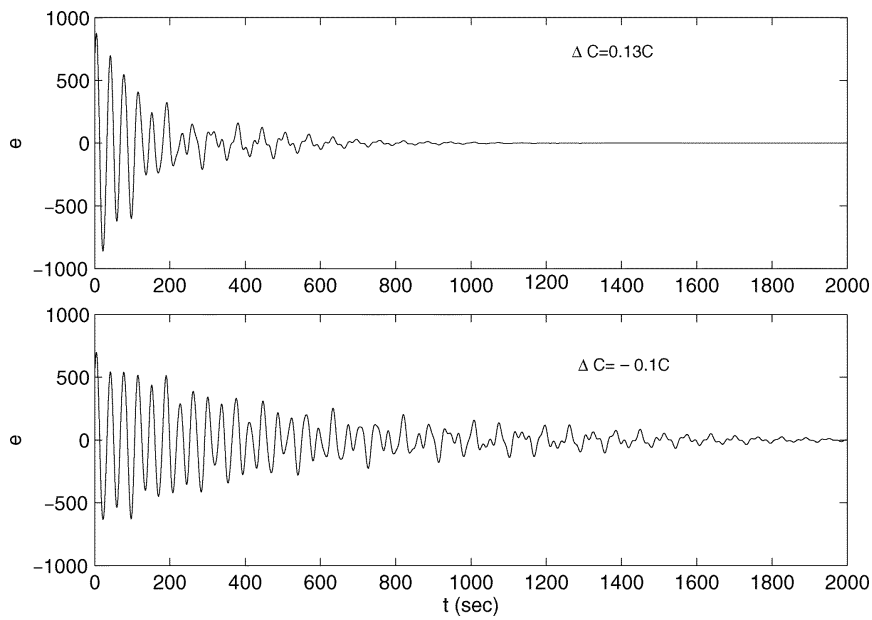

Fig. 12. Tracking errors under perturbed $C$, Case 2 .

Better robustness against measurement error can be achieved by reducing the observer gain. For example, if we place the eigenvalues of $\tilde{A}$ at

$$
-1.5 \pm 0.3 j,-1.5 \pm 0.6 j,-1.5 \pm 0.9 j,-1.5 \pm 1.5 j
$$

then the maximal elemment of $L$ is $-2.45 \times 10^{3}$ and output regulation can be achieved for $y=(C+\Delta C) x, \Delta C=k C$, with $|k| \leq 0.002$. Fig. 11 plots the traking errors under different measurement errors. The initial condition is the same as that in Fig. 10.

By choosing different $L$ and different $F_{2}$ for the feedback law $u=\operatorname{sat}\left(F_{2} v\right)$, the robustness against measurement error can be further enhanced, with possible degradation of transient performances. For instance, we choose $L$ to place the eigenvalues of $\tilde{A}$ at

$$
-1 \pm 0.2 j,-1 \pm 0.4 j,-1 \pm 0.6 j,-1 \pm 1 j
$$

and we choose

$$
F_{2}=\left[\begin{array}{ll}
-0.0056 & 0.0026
\end{array}\right]
$$

then output regulation can be achieved for $y=(C+\Delta C) x$, $\Delta C=k C$, with $k \in[0.9,1.13]$. Fig. 12 plots the tracking errors under different measurement perturbations. The initial condition is the same as that in Fig. 10. However, the convergence 
of the tracking error is much slower than that in Fig. 11. We have also carried out simulation with $\Delta C(t)=k_{0} \sin (\omega t) C$. The maximal $k_{0}$ that can be tolerated depends on $\omega$.

With a low observer gain, the parameter $T_{0}$ would be large and the initial states of the plant and exosystem have to be restricted in a region much smaller than the regulatable region. This shows a typical situation of trading the size of the set of initial conditions for robustness against parameter uncertainties.

From the simulation results, we see that there may exist conflict among the convergence performance, the robustness against model uncertainties and the size of the set of initial conditions. The feedback law and the observer gain have to be carefully designed to balance all these conflicting objectives. This problem will be further pursued in our future research.

\section{CONCLUSION}

In this paper, we have systematically studied the problem of output regulation for linear systems with bounded controls. The plants considered here are general and can be exponentially unstable. We first characterized the regulatable region, the set of initial conditions of the plant and the exosystem for which output regulation can be achieved. Based on given stabilizing state feedback laws, we then constructed state feedback laws and an error feedback law, that achieve output regulation on a subset of the regulatable region. The size of this subset depends on the domain of attraction under the given stabilizing state feedback law. The design method is demonstrated with an example and robustness issues are discussed through simulations. Further analysis on robustness will be pursued in our future study.

\section{REFERENCES}

[1] R. De Santis, "Output regulation for linear systems with antistable eigenvalues in the presence of input saturation," Int. J. Robust Nonlinear Control, vol. 10, pp. 423-438, 2000.

[2] R. De Santis and A. Isidori, "On the output regulation for linear systems in the presence of input saturation," IEEE Trans. Automat. Contr., vol. 46, pp. 156-160, Jan. 2001.

[3] Z. Ding, "Global output regulation of uncertain nonlinear systems with exogenous signals," Automatica, vol. 37, pp. 113-119, 2001.

[4] B. A. Francis, "The linear multivariable regulator problem," SIAM J. Control Optim., vol. 15, pp. 486-505, 1975.

[5] R. Freeman, "Global internal stabilizability does not imply global external stabilizability for small sensor disturbances," IEEE Trans. Automat. Contr., vol. 40, pp. 2119-2122, Dec. 1995.

[6] O. Hájek, Control Theory in the Plane. New York: Springer-Verlag, 1991.

[7] T. Hu and Z. Lin, Control Systems with Actuator Saturation: Analysis and Design. Boston, MA: Birkhäuser, 2001.

[8] — "Practical stabilization of exponentially unstable linear systems subject to actuator saturation nonlinearity and disturbance," Int. J. Robust Nonlinear Control, vol. 11, pp. 555-588, 2001.

[9] T. Hu, Z. Lin, and B. M. Chen, "An analysis and design method for linear systems subject to actuator saturation and disturbance," Automatica, vol. 38, no. 2, pp. 351-359, 2002.

[10] T. Hu, Z. Lin, and L. Qiu, "An explicit description of the null controllable regions of linear systems with saturating actuators," Syst. Control Lett., vol. 47 , no. 1 , pp. $65-78,2002$

[11] _ _ "Stabilization of exponentially unstable linear systems with saturating actuators," IEEE Trans. Automat. Contr., vol. 46, pp. 973-979, June 2001.

[12] T. Hu, Z. Lin, and Y. Shamash, "Semi-global stabilization with guaranteed regional performance of linear systems subject to actuator saturation," Syst. Control Lett., vol. 43, pp. 203-210, 2001.

[13] J. Huang, "Editorial of the special issue on output regulation of nonlinear systems," Int. J. Robust Nonlinear Control, vol. 10, pp. 321-322, 2000.

[14] _ , "Output regulation of nonhyperbolic nonlinear systems," IEEE Trans. Automat. Contr., vol. 40, pp. 1497-1500, Sept. 1995.
[15] J. Huang and W. J. Rugh, "On a nonlinear multivariable servomechanism problem," Automatica, vol. 26, pp. 963-972, 1990.

[16] A. Isidori and C. I. Byrnes, "Output regulation for nonlinear systems," IEEE Trans. Automat. Contr., vol. 35, pp. 131-140, Jan. 1990.

[17] J. Junkins, J. Valasek, and D. D. Ward, "Report of ONR UCAV Modeling Effort," Dept. Aerospace Eng., Texas A\&M Univ., College Station, TX, 1999.

[18] W. Lan and J. Huang, "Semiglobal stabilization and output regulation of singular linear systems with input saturation," IEEE Trans. Automat. Contr., vol. 48, pp. 1274-1279, June 2003.

[19] Z. Lin, Low Gain Feedback. London, U.K.: Springer-Verlag, 1998.

[20] Z. Lin and A. Saberi, "Semi-global exponential stabilization of linear systems subject to 'input saturation' via linear feedbacks," Syst. Control Lett., vol. 21, pp. 225-239, 1993.

[21] Z. Lin, A. A. Stoorvogel, and A. Saberi, "Output regulation for linear systems subject to input saturation," Automatica, vol. 32, pp. 29-47, 1996.

[22] J. Macki and M. Strauss, Introduction to Optimal Control. New York: Springer-Verlag, 1982

[23] E. D. Sontag, "An algebraic approach to bounded controllability of linear systems," Int. J. Control, vol. 39, pp. 181-188, 1984.

[24] R. Suarez, Alvarez-Ramirez, and J. Solis-Daun, "Linear systems with bounded inputs: Global stabilization with eigenvalue placement," Int. J. Robust Nonlinear Control, vol. 7, pp. 835-845, 1997.

[25] H. J. Sussmann, E. D. Sontag, and Y. Yang, "A general result on the stabilization of linear systems using bounded controls," IEEE Trans. Automat. Contr., vol. 39, pp. 2411-2425, Dec. 1994.

[26] A. R. Teel, "Feedback stabilization: Nonlinear solutions to inherently nonlinear problems," Ph.D. dissertation, Univ. California, Berkeley, CA, 1992.

[27] — "A nonlinear small gain theorem for the analysis of control systems," IEEE Trans. Automat. Contr., vol. 42, pp. 1256-1270, July 1996.

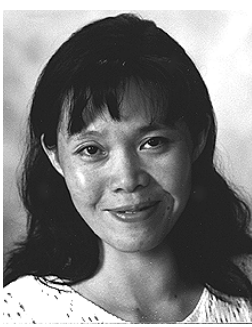

Tingshu Hu(S'99-SM'01) received the B.S. and M.S. degrees in electrical engineering from Shanghai Jiao Tong University, Shanghai, China, in 1985 and 1988 , respectively, and the Ph.D. degree in electrical engineering from University of Virginia, Charlottesville, in 2001

She has been a Postdoctoral Researcher at University of Virginia and the University of California, Santa Barbara. Her research interests include nonlinear systems theory, optimization, robust control theory, and control application in mechatronic systems and biomechanical systems. She is a coauthor of the book Control Systems with Actuator Saturation: Analysis and Design (Boston, MA: Birkhäuser, 2001).

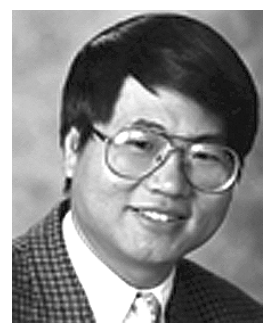

Zongli Lin (S'89-M'90-SM'98) received the B.S. degree in mathematics and computer science from Xiamen University, Xiamen, China, in 1983 , the M.Eng. degree in automatic control from the Chinese Academy of Space Technology, Beijing, China, in 1989, and the Ph.D. degree in electrical and computer engineering from Washington State University, Pullman, WA, in 1994.

$\mathrm{He}$ is currently an Associate Professor with the Charles L. Brown Department of Electrical and Computer Engineering, the University of Virginia, Charlottesville. Previously, he has worked as a Control Engineer with the Chinese Academy of Space Technology and as an Assistant Professor with the Department of Applied Mathematics and Statistics at State University of New York, Stony Brook. His current research interests include nonlinear control, robust control, and modeling and control of magnetic bearing systems. In these areas, he has published several papers. He is also the author of the book Low Gain Feedback (London, U.K.: Springer-Verlag, 1998), a coauthor of the book Control Systems with Actuator Saturation: Analysis and Design (Boston, MA: Birkhäuser, 2001) and a coauthor of the book Linear Systems Theory: A Structural Decomposition Approach (Boston, MA: Birkhäuser, 2004).

Dr. Lin served as an Associate Editor of IEEE TRANSACTIONS ON AUTOMATIC CONTROL from 2001 to 2003, and is currently an Associate Editor of Automatica. He is a Member of the IEEE Control Systems Society's Technical Committee on Nonlinear Systems and Control and heads its Working Group on Control with Constraints. He was the recipient of a U.S. Office of Naval Research Young Investigator Award. 